Portland State University

PDXScholar

Spring 7-6-2016

\title{
Anachronisme, rebuts et survivances dans Les escaliers de Chambord et le Dernier Royaume de Pascal Quignard
}

Etienne Lussier

Portland State University

Follow this and additional works at: https://pdxscholar.library.pdx.edu/open_access_etds

Part of the French and Francophone Language and Literature Commons Let us know how access to this document benefits you.

\section{Recommended Citation}

Lussier, Etienne, "Anachronisme, rebuts et survivances dans Les escaliers de Chambord et le Dernier Royaume de Pascal Quignard" (2016). Dissertations and Theses. Paper 3037.

https://doi.org/10.15760/etd.3032

This Thesis is brought to you for free and open access. It has been accepted for inclusion in Dissertations and Theses by an authorized administrator of PDXScholar. Please contact us if we can make this document more accessible: pdxscholar@pdx.edu. 
Anachronisme, rebuts et survivances dans Les escaliers de Chambord et le Dernier Royaume de Pascal Quignard

by

Étienne Lussier

A thesis submitted in partial fulfillment of

the requirements for the degree of

\title{
Master of Arts
}

in

French

\author{
Thesis Committee: \\ Annabelle Dolidon, Chair \\ Stephen Walton \\ Gina Greco
}

Portland State University

2016 


\begin{abstract}
This thesis investigates the particular conception of time developed by Pascal Quignard in his series Dernier Royaume and his novel Les escaliers de Chambord. We argue that this conception of time, which Quignard refers to as Le Jadis, operates through an anachronistic method based on a semiotic apparatus of waste, or rejects. The argument is presented in three different chapters. The first chapter uses the work of Jacques Rancière and Georges Didi-Huberman to show the way in which the concept of anachronism embraces a temporality that blurs the lines between past, present, and future and creates openings to summon and redeem forgotten relics of the past. The second chapter analyzes this open relation to time in Dernier Royaume and Les escaliers de Chambord. In these works, Quignard's notion of Le Jadis seeks specifically to awaken a vertiginously distant conception of past that is incomplete, non-linear, and perpetually becoming. The last chapter analyzes the manner in which Le Jadis can reappear as an active force through the mediation of waste, which constitutes a specific class of signs. The work of Quignard is one of montage and remontage — fragment by fragment, scrap by scrap - of the pieces of memory, which he strives to reopen and release from the linear entrapment of chronological time to which they have been relegated.
\end{abstract}




\section{TABLE DES MATIÈRES}

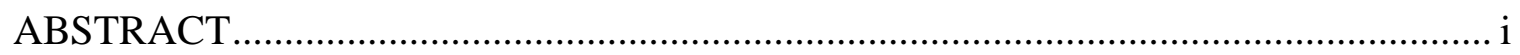

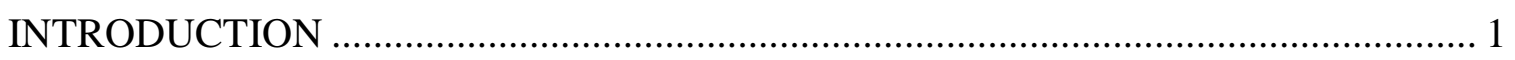

\section{CHAPITRE 1}

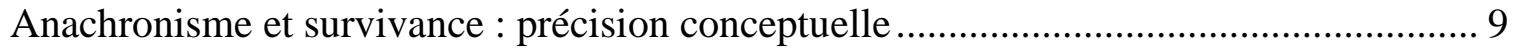

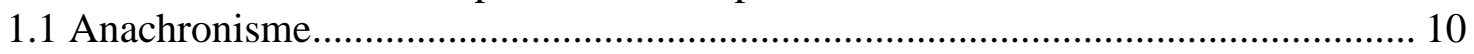

1.2 La survivance et le symptôme ......................................................................... 14

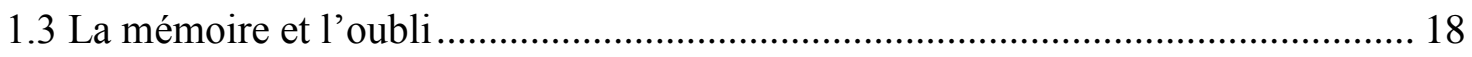

\section{CHAPITRE 2}

Le Jadis: Conceptualisation du temps dans Dernier Royaume ..................................... 20

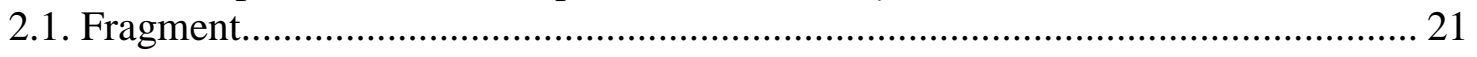

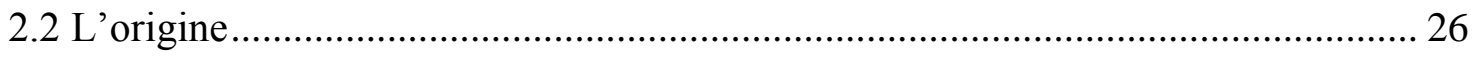

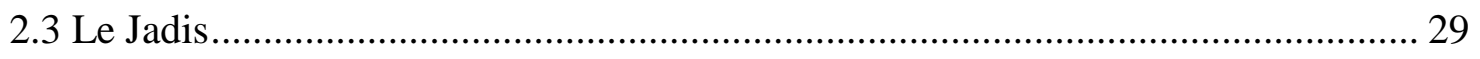

$2.4 \mathrm{La}$ perte

\section{CHAPITRE 3}

Les rebuts et les Sordidissimes dans Les Escaliers de Chambord ................................... 36

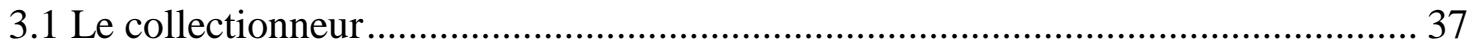

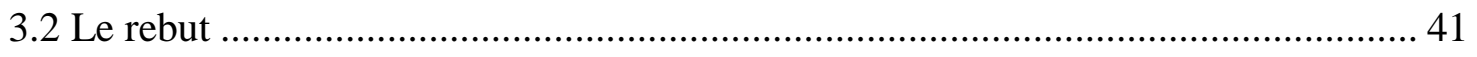

3.3 Des rebuts physiques aux rebuts de la mémoire ............................................. 47

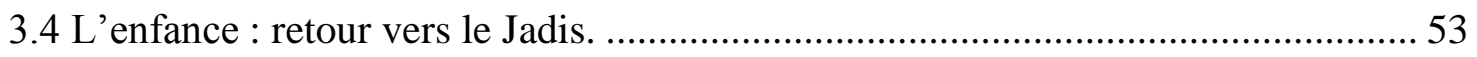

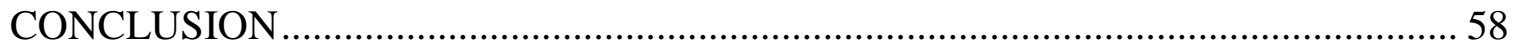

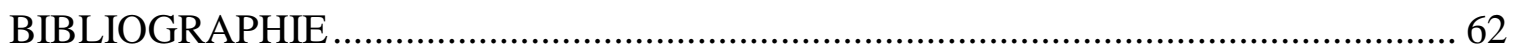




\section{INTRODUCTION}

On présente souvent Pascal Quignard comme étant l'une des figures incontournables de la littérature française contemporaine. Si Quignard fait partie du paysage littéraire français depuis la deuxième moitié des années 1970, soit depuis la publication de son premier roman intitulé Le lecteur (1976), nous pouvons néanmoins nous questionner sur le rapport qu'entretient Quignard avec l'ensemble du champ littéraire français. De qui et de quoi Quignard est-il le contemporain ? Et que signifie réellement être «contemporain »? Si l'usage trivial du terme renvoie à ce qui se situe dans le présent, dans l'actualité et la simultanéité du temps-même, aborder l'œuvre de Quignard nécessite néanmoins de se soustraire à une périodisation trop stricte qui épouserait de trop près les plis du temps présent. Le philosophe Giorgio Agamben, reprenant les écrits de Roland Barthes, remarquait à quel point la valeur de contemporanéité d'une œuvre dépend en réalité de son inactualité. Agamben écrit :

Celui qui appartient véritablement à son temps, le vrai contemporain, est celui qui ne coïncide pas parfaitement avec lui ni n'adhère à ses prétentions et se définit en ce sens, comme inactuel; mais précisément pour cette raison précisément par cet écart et cet anachronisme, il est plus apte que les autres, à percevoir et à saisir son temps. («Qu’est-ce que le contemporain »20)

La tâche de l'écrivain, du poète ou de l'artiste réellement contemporain est donc de se mettre à distance, de se décaler, voire de tourner le dos aux «lumières » du temps présent pour plonger au cœur des ténèbres, des zones d'ombre, que nous offre le temps présent. 
Le contemporain se désolidarise de son époque pour mieux l'observer : son rapport au temps est anachronique.

L'œuvre de Pascal Quignard est exemplaire de ce rapport au temps ambivalent traversé par des pointes d'anachronismes. Son œuvre romanesque traverse les époques, les styles, les genres : Quignard se positionne systématiquement en retrait et en décalage avec le temps présent. Mais ce souci littéraire est aussi accompagné d'un souci théorique. Depuis la parution de ses Petits traités au début des années 1980, Quignard élabore un temps qui serait tout autre, un temps hors du Chronos $^{1}$, radicalement inorienté et ouvert : un temps déréglé qui échappe au pur présent et à la linéarité du temps historique. Cette temporalité, Quignard l'appelle le Jadis.

En ce sens, Quignard retrouve une filiation intellectuelle ayant mis l'«anachronisme » au centre de ses préoccupations et qui semble aujourd'hui connaître un certain renouveau en France, notamment avec les travaux de Georges Didi-Huberman sur Aby Warburg et Walter Benjamin². Parler d'anachronisme pour qualifier l'œuvre de Quignard n'est pas anodin. Chez des historiens de l'art comme Georges Didi-Huberman et Aby Warburg, nous retrouvons une véritable heuristique, voire une épistémologie de l'anachronisme, où les images doivent être perçues comme des « objets temporellement impurs, complexes, surdéterminés » (Didi-Huberman, Devant le temps 22). DidiHuberman poursuit :

\footnotetext{
${ }^{1}$ Par temps du chronos, nous entendons, avec Gilles Deleuze, un temps qui se déploie comme «mouvement réglé des présents vastes et profonds» (191), du temps présent limité — et circulaire — de « l'action des corps et des causes » (190). Voir Logique du sens. Paris: Éditions de Minuit, 1969.

${ }^{2}$ On peut remonter cette tradition jusqu'aux Considérations inactuelles de Friedrich Nietzsche pour qui il était important d'agir en décalage avec le temps afin «d'exercer une influence inactuelle, c'est-à-dire d'agir contre le temps, donc sur le temps, et . . . au bénéfice d'un temps à venir». Considérations inactuelles. Paris : Gallimard, 1990, p. 94.
} 
l'anachronisme semble émerger à la pliure exacte du rapport entre image et histoire : les images, certes, ont une histoire ; mais ce qu'elles sont, le mouvement qui leur est propre, leur pouvoir spécifique, tout cela n'apparaît que comme un symptôme - un malaise, un démenti plus ou moins violent, une suspension — dans l'histoire. (25)

L'anachronisme n'est donc pas une faute méthodologique comme le conçoit la perspective historiographique ${ }^{3}$, mais un outil permettant de monter et remonter des symptômes survivants de l'inconscient - visuel chez Didi-Huberman — et de l'imaginaire. Il y a chez Quignard une pensée analogue qui procède par amoncellement de symptômes et de survivances.

Cette temporalité anachronique, nous souhaitons l'aborder en prenant les œuvres où Pascal Quignard s'est adressé directement à la question du temps. Depuis 2002, Quignard a amorcé un cycle intitulé Dernier Royaume, réunissant jusqu'à ce jour plus de neuf titres. Cette série, dont les trois premiers tomes parus en 2002 furent couronnés du prix Goncourt, développe une conception de la temporalité anachronique autour de cette notion de Jadis. Le matériau qui y est déployé s'avère décontenançant en raison de sa forme. Quignard y mélange réflexions personnelles, philosophiques, contes, anecdotes historiques et étymologiques, ainsi que divers fragments fictionnels hybrides venant brouiller toutes limites entre les genres. Quignard poursuit ici un travail qui avait déjà été amorcé dans ses Petits traités, où ce dernier accumulait nombre de fragments qui échappent aux normes de catégorisation littéraire. Sous cette apparence bigarrée de fragments éclectiques se cache non seulement un geste esthétique, mais aussi une exigence épistémologique visant à sauvegarder un savoir laissé en jachère dans la mémoire et à le restituer, à le re-monter. Comme Guillaume Asselin le souligne :

\footnotetext{
${ }^{3}$ La première section de notre premier chapitre traitera de cette question plus en profondeur.
} 
Théâtre des permutations et des transmutations, le Dernier Royaume est le lieu d'une hybridation sans limites. Chaque objet y impose sa propre règle d'exposition. . . . Ainsi Quignard parle-t-il des objets qui tracent les contours de son Dernier Royaume comme de «sèmes asèmes » et de «dépôt sématique» sans signification qu'on ne peut que remuer. La logique de l'excès, qui préside au collectionnisme sous l'espèce d'une énumération dont l'essence consiste à démembrer ce qu'elle assemble, conduit, à terme, à démotiver les signes, à les retirer du réseau symbolique qui les tient captifs du sens pour leur redonner une véritable positivité d'objets réels. (12)

Cette collection de pensées sauvages, plus qu'un amas de débris épars, sous-tend une conception sémiotique où le désordre acquiert une valeur positive permettant de démonter et de remonter des morceaux de mémoire appartenant au passé. Dernier Royaume rejoint alors cette conception anachronique du montage. Cette démarche, plutôt que de rechercher une vérité figée ou une compréhension certaine, prêche par excès. Sa force réside plutôt dans son indétermination.

Le travail quignardien dans le Dernier Royaume consiste à remonter, fragment par fragment, déchet par déchet, les morceaux du passé, et à les ré-ouvrir, à les «sortir »du piège linéaire du temps chronologique auquel ils avaient été assignés. La forme du Dernier Royaume, rencontre alors le fond: les fragments du passé sont appelés à rencontrer le Jadis. C'est ainsi que Quignard dans Dernier Royaume juxtaposera, côte à côte, des réflexions sur la poésie antique japonaise de l'époque Heian d'Izumi Shikibu à des réflexions de Bossuet, Montaigne ou encore Hannah Arendt. En évoquant ces pensées enfouies dans la mémoire occidentale et orientale, Quignard construit une pensée sur la temporalité en s'aidant de fragments eux-mêmes dispersés dans le temps et décontextualisés. 
Ce travail de «chiffonnier», comme le dirait Walter Benjamin ou encore Siegfried Kracauer, mis en place dans Dernier Royaume, se retrouve aussi dans l'œuvre romanesque de Quignard. Nous avons décidé de l'illustrer dans son roman de 1989 intitulé Les escaliers de Chambord, un roman aux aspects formels plutôt « classiques », dans lequel Quignard met en scène le personnage d'Edouard Furfooz ${ }^{4}$ qui fait figure de «chiffonnier». Furfooz, antiquaire de profession, collectionneur de jouets anciens, se voit emporté dans un tumulte labyrinthique. Constamment en déplacement entre divers pays de l'Europe et les États-Unis, Furfooz est un personnage déraciné dans une quête insatiable d'objets rares : l'acte de collection est son leitmotiv, et les jouets agissent pour lui comme autant de traces originaires. Au fil du récit, passivement, Quignard établit un jeu de piste, une chasse indiciaire marquée par divers rebuts avec lesquels il tentera de reconstituer un traumatisme originaire remontant à l'enfance. Fragment par fragment, trace mnésique par trace, Furfooz est confronté à une panoplie de signes, de survivances, dont il s'avère incapable de reconstruire le fil d'Ariane. C'est ainsi que la découverte d'un rebut, soit une petite barrette bleue en forme de grenouille, agira comme un

\footnotetext{
${ }^{4}$ Le patronyme de Furfooz renvoie à un site archéologique belge du même nom où fut découverte une grotte ayant servi de lieu de sépulture et datant d'environ -20000 avant notre ère. Nous retrouvons dans l'ensemble de l'œuvre de Quignard une certaine volonté «archéologique», voire «archéo-pathique» pour le dire comme Dominique Viart, où la scène primitive anthropologique est appelée à resurgir du passé. Viart écrit : «le récit quignardien serait une archéo-logie. Ou plutôt, car il ne s'agit pas de construire un système logique de l'archè, mais d'éprouver et de faire éprouver ce que l'exercice de la raison efface et censure : une archéo-pathie. À l'érudition se combine en effet parfois la fascination empathique» (30). Ce pathos, comme Viart le souligne, apparaît comme une célébration de l'origine et de l'archaïque. Dans un passage de Rhétorique spéculative, Quignard écrit: «Entrez dans Lascaux. Entrez dans Niaux. Entrez dans les grottes de Pair-non-Pair et de Gargas. Entrez au Font-de-Gaume, à Madeleine, à Lespugue. Entrez dans le Cellier ou à Vogelherd. Entrez dans Oreille-d'Enfer» (211).
} 
déclencheur de mémoires lançant une chasse aux traumatismes refoulés. Le rebut, ainsi, apparait comme un signe permettant aux survivances de finalement refaire surface.

Les escaliers de Chambord pose une triple réflexion sur le temps, l'espace et la trace. Nous montrerons que la temporalité du Jadis fait irruption dans le roman comme ce temps de l'antériorité invisible qui rythme le développement de l'intrigue. Le sens résiste à son dévoilement. Cette temporalité n'est donc pas celle d'un passé figé et fermé, mais sur une signification ouverte toujours en devenir. À ce trouble du temps se superpose un trouble de l'espace. L'impossibilité du personnage à se positionner — ou se figer — dans le temps trouve son corrélat dans l'espace : le Jadis, après tout, est bien une forme de nomadisme. Furfooz n'a aucun domicile fixe; il parcourt compulsivement l'Europe et l'Amérique à la recherche d'antiquités et de rebuts délaissés, qu'il accumule sachant très bien la difficulté qu'il aura à trouver preneur. Le geste de Furfooz, et de Quignard, est donc celui de l'antiquaire: celui qui cherche à démonter de manière erratique les souvenirs qui font crise, laissant parfois planer le doute à savoir si ceux-ci sont bien réels ou fantasmés.

Dans le présent travail, nous analyserons comment la conception temporelle du

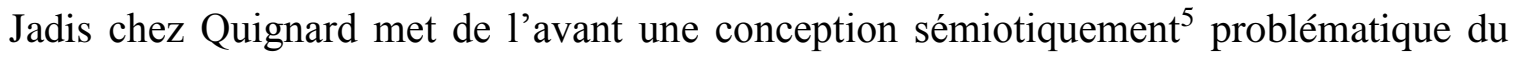
rebut, de l'histoire et du temps, et nous chercherons à comprendre comment cela se traduit dans son œuvre romanesque. Entre l'écriture fragmentaire et le roman, le geste d'écriture quignardien demeure le même : restituer, relire et remonter des morceaux de mémoire et de temps. Notre démarche s'inscrit alors dans une tentative de mettre en

${ }^{5}$ Il ne s'agit pas ici de parler de la sémiologie ou de la sémiotique dans une perspective strictement linguistique et structurale. Concevoir les rebuts dans l'œuvre littéraire de Quignard comme des signes implique ici de les traiter comme s'il s'agissait d'objets matériels et tangibles ayant une existence réelle. 
relation des œuvres qui sont complètement différentes d'un point de vue de la forme et de souligner la récurrence thématique qui anime l'œuvre quignardienne. En effet, tant la question du temps que la question du fragment traversent l'ensemble de l'œuvre. La question à laquelle nous tenterons de répondre s'énonce ainsi : comment est-ce que le rebut — ou le déchet - participe chez Quignard à une quête nous permettant de rejoindre une temporalité singulière et anachronique? Nous démontrerons que dans les œuvres à l'étude il y a un rapport au temps qui se conçoit comme un temps anachronique. Par l'entremise du rebut, nous croyons trouver un outil sémiotique qui nous permettra de retrouver cette temporalité originaire que Quignard a désignée sous le concept du Jadis. Cette «logique » du rebut apparaît tant dans la forme du Dernier Royaume que dans le fond de l'œuvre romanesque quignardienne, au point où le rebut acquiert la fonction de structurer l'intrigue.

L'argument se déploiera en trois temps. Dans notre premier chapitre, nous apporterons des précisions d'ordre conceptuel. Le concept d'anachronisme est chargé d'une multitude de connotations que nous devons neutraliser. L'anachronisme ne peut se résumer à une simple erreur dans la chronologie des évènements. Il s'agira ici de montrer comment ce terme a un versant positif nous permettant d'entrevoir une relation au temps historique différente. Avec les travaux de Georges Didi-Huberman, nous aborderons deux autres concepts, soit ceux de la survivance et du symptôme. Avec cette triade conceptuelle — anachronisme, survivance et symptôme —, nous serons en mesure de mieux cerner les enjeux que comporte la notion de Jadis développée dans le Dernier Royaume, mais aussi de dégager des outils pour analyser le roman Les escaliers de 
Chambord. Notre deuxième chapitre sera consacré directement à la notion de Jadis développée par Quignard. Nous analyserons la méthode fragmentaire d'écriture qu'il met de l'avant dans le Dernier Royaume et comment cette démarche permet de rejoindre et de déployer une conception originale et originaire du temps. Finalement, dans notre dernier chapitre, nous aborderons la thématique du rebut et du déchet. Les rebuts sont des signes, porteurs de traces spécifiques, qui montrent une transformation dans leur destination et leur emploi. Ils constituent une classe « sémiotique » particulière qui, pour le dire comme Krzysztof Pomian, « réunit tout ce que les hommes abandonnent, évacuent ou détruisent » (82). Nous retrouvons tant dans le quatrième tome du Dernier Royaume intitulé Sordidissimes que dans Les escaliers de Chambord une riche réflexion sur la question du rebut. Nous verrons comment l'acte de collection compulsif et le «remontage» des souvenirs de l'enfance sont alors autant d'ouvertures du passé susceptibles de créer ou de réveiller le Jadis. 


\section{CHAPITRE 1}

\section{Anachronisme et survivance : précision conceptuelle}

Avant d'aborder l'œuvre de Pascal Quignard et la conception singulière du temps qu'il développe, il importe d'apporter quelques précisions d'ordre terminologique. Dans ce premier chapitre, nous allons préciser la portée théorique et conceptuelle de l'anachronisme. Ce concept qui possède diverses significations ne se limite pas qu'à un problème d'ordre «méthodologique »: il interroge radicalement la question du temps, de la mémoire et de l'histoire. Nous tenons à dégager avec cette notion un rapport au temps et à l'histoire qui ne se restreint pas à une conception historiographique, c'est-à-dire qui dépasse le savoir officiel promu par la démarche historienne. L'anachronisme est donc un concept qui tente de bouleverser les certitudes du savoir construit par les historiens. Comme il en sera question dans notre deuxième chapitre, la temporalité que développe Quignard dans Sur le jadis, sera elle-même investie de ce pouvoir de «transgression ». Par la suite, ce concept d'ordre temporel permettra d'introduire deux autres notions qui résonneront dans l'œuvre de Quignard: la survivance et le symptôme. Le symptôme est la trace de survivances passées qui ressurgissent dans le présent, et vient déchirer tant la représentation que le temps. Ces deux concepts, intimement liés à la question de l'anachronisme, posent les premiers jalons d'une vision sémiotique qui place le rebut au cœur de processus de signification inconscients. La question du rebut — compris comme un objet temporel trouble essentiellement anachronique — sera fondamentale pour analyser Les escaliers de Chambord. 


\subsection{Anachronisme}

«Qu'est-ce que le temps? Si personne ne me pose la question, je sais; si quelqu'un me pose la question et que je veuille expliquer, je ne sais plus » (qtd. dans Ricœur, Temps et récit 1 25). Cette citation de Saint-Augustin nous met en garde, lorsque vient le temps de définir des termes et des concepts, que parfois les choses les plus proches de nous sont en réalité les plus difficiles à cerner. Ce problème apparaît lorsque nous questionnons le temps : celui-ci semble vouloir perpétuellement nous glisser entre les doigts ; il est à proprement parler insaisissable, bien que nous en fassions l'expérience dans chacune des sphères de notre vie. Dans le premier tome de Temps et récit, Paul Ricœur, en revenant sur la conception saint-augustinienne du temps, procède à une fine analyse de la question de la temporalité. Ricœur démontre qu'il y a chez Saint-Augustin une réflexion sur le temps qui permet de ramener dans le présent les catégories de passé (la mémoire) et de futur (l'attente), et où chacune de ces catégories combinées interagissent les unes avec les autres. Elles sont distendues les unes des autres. Le temps, qui pour Saint-Augustin est une distension de l'âme, une distantio animi, se doit alors d'être envisagé comme une discordance entre différents moments hétérogènes.

Concevoir le temps comme discordance rend possible une vision de la temporalité tout autre que celle du temps «mécanique», c'est-à-dire celle du temps homogène et vide, sans forme, correspondant au mouvement de l'aiguille qui parcourt la circonférence du cadran. Le temps devient hétérogène, et les catégories de passé, de présent et de futur sont dorénavant susceptibles de cohabiter. Comment parler d'une 
cohabitation entre le moment passé et le présent? L'anachronisme, qui désigne cette discordance de moments hétérogènes, est un principe fondamental pour comprendre la connexion entre l'histoire, le passé et la mémoire. Comme l'étymologie du terme le suggère, anachronisme vient du grec ancien et est composé du préfixe - ana qui veut dire entre autres avant, ou en arrière, et du terme Khronos qui signifie temps. Dans son usage premier, l'anachronisme désigne donc l'«action de placer un fait, un usage, un personnage, etc., dans une époque autre que l'époque à laquelle ils appartiennent ou conviennent réellement » (Grand Robert 344). Cette première définition du Grand Robert souligne la dimension historique de ce concept et engage un questionnement d'ordre méthodologique et épistémologique sur la vérité de l'histoire. L'anachronisme, pris dans cette perspective, devient «le péché mortel de l'histoire» (Robin 52), un obstacle majeur à la constitution d'une science historique. Cette idée de «péché capital» de la science historique sera notamment partagée par Lucien Febvre et l'École des Annales, école qui eut une place prédominante au $\mathrm{XX}^{\mathrm{e}}$ siècle dans le champ historiographique français. Autrement dit, l'anachronisme est une faute liée à la chronologie des évènements, des faits, des pensées: il concerne le temps homogène et linéaire «des dates». L'anachronisme est donc cette erreur méthodologique à laquelle les historiens se doivent de ne pas succomber, au risque de remettre en question le statut de scientificité de leur discipline.

Mais dans son article «Le concept d'anachronisme et la vérité en histoire», le philosophe Jacques Rancière démontre qu'une véritable conceptualisation de l'anachronisme ne peut s'en tenir à cette stricte perspective historiographique. Ce 
concept, afin de véritablement prendre son «envol», doit quitter les considérations méthodologiques et épistémologiques sur le statut de la vérité de l'Histoire, pour déborder vers une conception ontologique et poétique. La question de l'anachronisme rejoint la poétique dans la mesure où les faits historiques doivent être saisis comme une disjonction entre l'énonciation, le passé et la vérité du passé. Pour Rancière, la définition classique d'anachronisme — telle que comprise par la science historique — est antihistorique dans la mesure où elle rend impossible tout mouvement de l'histoire et toute historicité. Les idées, les conceptions du monde ne sont jamais en parfaite adéquation avec leur époque, contrairement à ce que soutiennent certains historiens. Le mouvement de l'histoire survient lorsque des idées et des acteurs parviennent à s'échapper de l'emprise du présent et à être en déphasage avec leur époque. Il y a donc un versant positif à l'anachronisme : il ouvre l'histoire et la met en mouvement.

Selon Rancière, «il y a d'histoire pour autant que les hommes ne "ressemblent" pas à leur temps, pour autant qu'ils agissent en rupture avec leur "temps", avec la ligne de temporalité qui les met à leur place » (66). Le temps «historiographique » se referme sur lui-même, n'admettant aucune ouverture ou production de «nouveauté ». Rancière propose donc une conception sérielle du temps où la ligne temporelle se décuple en une multiplicité de possibilités offrant différents modes de connexion. L'anachronisme crée des ouvertures : «il y a des modes de connexion que nous pouvons appeler positivement des anachronies : des évènements, des notions, des significations qui prennent le temps à rebours, qui font circuler le sens d'une manière qui échappe à toute contemporanéité, à toute identité du temps avec lui-même»(67-68). Cette conception permet d'ouvrir un 
temps inédit, d'ouvrir l'histoire, de la déconstruire et d'en montrer son impureté, c'est-àdire d'offrir des «connexions » avec les différentes lignes de temporalité.

On touche ici à la question de la poétique. Comme le suggère Rancière, la pratique historienne partage d'emblée une connexion avec la poésie en tant que ces deux « disciplines », ou régimes de vérité, se constituent de manière analogue. Rancière écrit : «La promotion de l'histoire comme discours de vérité passe par sa capacité de se faire semblable à la poésie, d'imiter pour son compte les puissances de la généralité poétique » (56). Déjà, dès les années 1960, Roland Barthes avait tenté de démontrer comment les discours d'ordre historique et poétique partageaient nombre de caractéristiques similaires. Barthes écrit :

... l'entrée de l'énonciation dans l'énoncé historique ... a moins pour but de donner à l'historien une possibilité d'exprimer sa «subjectivité", comme on le dit communément, que de "compliquer le temps chronique » de l'histoire en l'affrontant à un autre temps, qui est celui du discours luimême et que l'on pourrait appeler par raccourci le temps-papier; en somme la présence, dans la narration historique, de signes explicites d'énonciation viserait à «déchronologiser» le «fil» historique et à restituer, ne serait-ce qu'à titre de réminiscence ou de nostalgie, un temps complexe, paramétrique, nullement linéaire, dont l'espace profond appellerait le temps mythique des anciennes cosmogonies, lié lui aussi à la parole du poète ou du devin. ${ }^{6}$ (Barthes 68)

Non seulement le discours historique apparaît bel et bien comme un discours construit d'intrigues, mais celui-ci, comme Barthes le souligne, joue sur des registres temporels «déchronologisants», convoquant aussi un registre qui se déploie dans l'ordre de la mémoire. L'histoire est faite de jeux rythmiques entre des chronologies et des anachronies, d'un travail de montage distendu entre l'écriture du présent et de la

\footnotetext{
${ }^{6}$ Pour l'instant, il est important de garder à l'esprit que l'acte d'écriture et d'énonciation chez Quignard doit se comprendre comme une tentative de transformer ce qui fait symptôme et de rompre avec le temps chronologique.
} 
mémoire, ou pour le dire comme Georges Didi-Huberman, d'une déconstruction du récit « en zig-zag, en changement de tempi, en complexité non-linéaire, en frottement de temps hétérogène » (Devant le Temps 38).

À la lumière de ces différents éléments, nous pouvons alors concevoir l'anachronisme non pas comme ce «mal suprême» historiographique, comme ce tort méthodologique, mais davantage comme une donnée essentielle à tout travail historique, voire à toute réflexion mettant au centre de ses préoccupations la question du temps historique. C'est ainsi que nous pouvons avancer l'idée avec Didi-Huberman qu' « il n'y a d'histoire qu'anachronique » (Devant le Temps 39). L'anachronisme est cette note discordante qui aide à combattre la conception mécanique et vide du temps.

\subsection{La survivance et le symptôme}

Jusqu'à présent, nous avons envisagé l'anachronisme comme un concept qui met en jeu le triple rapport du temps, de l'histoire et de la vérité, autrement dit, qui interroge la non-coïncidence entre des actions, des pensées, des évènements et leur époque. Nous avons vu avec Jacques Rancière que cette conception de l'anachronisme n'est pas suffisante en elle-même et qu'on ne peut la restreindre à une simple erreur de chronologie d'ordre historiographique. Bien que Rancière ne développe pas de véritable heuristique de l'anachronisme, nous pouvons néanmoins commencer à percevoir un versant «positif » à cette notion. C'est cette idée qui anime une partie de l'œuvre de l'historien de l'art Georges Didi-Huberman. Comme Didi-Huberman l'écrit : «L'anachronisme, dès lors, pourrait ne pas être réduit à cet horrible péché qu'y voit spontanément tout historien 
patenté. Il pourrait être pensé comme un moment, comme un battement rythmique de la méthode, fût-il son moment de syncope »(Devant le Temps 21-22). Comment ouvrir cette notion? Le Grand Robert nous fournit une deuxième définition qui nous aidera à amorcer un décloisonnement, ou une ouverture du concept d'anachronisme. On peut concevoir l'anachronisme «[c]omme décalage dans le temps . . . ce qui est ... périmé, désuet; chose, usage, imitation anachronique. Survivance» («Anachronisme» 344). Premièrement, cette définition reprend l'idée de la non-coïncidence des temps, de la coexistence d'éléments qui ne sont pas contemporains. Deuxièmement, l'anachronisme intègre ce qui est périmé ou désuet, c'est-à-dire ce qui ne coïncide plus parfaitement avec son époque, ce qui se distancie du contemporain, ou comme le disait Rancière, de ce qui est en «rupture » avec son temps. Finalement, cette définition introduit l'étonnante notion de survivance. Cette notion, qui apparaît conjointement avec l'anachronisme, nous donne une autre perspective sur le temps historique.

L'idée même de survivance renvoie à ce qui subsiste au passage du temps : le passé n'est pas une chose révolue - dont l'historien pourrait s'attrister de la perte définitive —, mais quelque chose qui survit, qui revient malgré sa disparition. La lecture faite par Georges Didi-Huberman des travaux d'Aby Warburg constitue l'un des terreaux les plus fertiles pour tenter de bien délimiter cette notion directement liée à la question d'anachronisme. Dans l'œuvre de l'historien de l'art Aby Warburg, nous retrouvons deux notions clefs, soit la notion de survivance (Nachleben) et celle des formules de pathos (Pathosformel), qui permettent d'approfondir et d'ouvrir le temps historique. Chez Warburg, la notion de Nachleben renvoie à ces «puissances de la mémoire » et à ces 
«potentialités du désir», tandis que «la notion de Pathosformel permet, quant à elle, d'observer au vif des gestes, des symptômes, des images » (Didi-Huberman, Atlas 108). La survivance ne saurait ici nous rassurer quant à une orientation déterminée du passé ou du devenir dans le temps historique. La survivance est plutôt un symptôme — au sens psychanalytique du terme ${ }^{7}$ - porteur de désorientation temporelle, et plutôt qu'en simplifier sa représentation, elle en complexifie radicalement sa structure en y privilégiant la survivance anachronique des formes. Pour résumer plus concrètement la démarche «historienne» de Warburg, ce qui importe est de délier le sort réservé aux images en tentant de remonter la trace des images — qui somnole dans le temps —, et de retrouver leurs apparitions intermittentes dans l'histoire. Comme le souligne DidiHuberman :

la survivance suppose tout un ensemble d'opérations où jouent de concert l'oubli, la transformation de sens, le souvenir provoqué, la retrouvaille inopinée, etc. - cette complexité devant nous rappeler le caractère culturel, non naturel de la temporalité en jeu.... [U]n jeu des «pauses » et des «crises », des « sauts » et des « retours périodiques »..., tout cela qui forme, non un récit de l'histoire, mais un écheveau de la mémoire. (Image survivante 92)

Le temps historique apparaît alors dans ces latences, dans ces crises, dans ces contretemps et dans ces symptômes qui révèlent la nature fantomale des formes plastiques dans le temps. ${ }^{8}$ Autrement dit, la fonction première de cette notion de

\footnotetext{
${ }^{7}$ Il faut comprendre le symptôme au sens freudien du terme, c'est-à-dire comme une actualisation d'une mémoire qui est inconsciente, qui se déplace, qui est mouvante et qui se situe au centre de désirs contradictoires. Nous y reviendrons.

${ }^{8}$ Par exemple Warburg analysait des œuvres picturales du Quattrocento, et plutôt que de simplement en faire une étude d'ordre iconographique (cherchant à déterminer par exemple qui sont les personnages représentés, quelle est la signification des symboles dissimulés dans l'œuvre, etc.), il essayait de trouver des motifs anachroniques cachés dans les petits détails des œuvres. C'est ainsi qu'il chercherait plutôt des
} 
survivance est d'anachroniser l'histoire et le présent. S'il y a eu disparition, le modèle de la «survivance» tente alors de remonter les traces de cet oubli et de se rendre «capable d'une mémoire, d'un retour, voire d'une renaissance » (Image Survivante 89). Ce qui fut oublié n'a donc pas été perdu à tout jamais, mais a disséminé dans le temps des traces, des indices de son passage. Celles-ci demeurent partiellement récupérables, à condition d'être attentif aux signes, aux manifestations symptomatiques.

Si pour Warburg — et par extension pour Didi-Huberman — la notion de survivance s'applique principalement à un savoir du symptôme visuel, nous pensons pouvoir l'élargir et en dégager une perspective sémiotique qui pourrait s'appliquer au signe en général. Ainsi, pour saisir la manifestation concrète des survivances, il faut les rattacher à un type de signe susceptible de pointer vers les latences de l'histoire. C'est ainsi qu'il nous faut revenir sur la notion de symptôme. Le symptôme est «ce moment où viennent débattre et s'entrelacer le présent du pathos et le passé de la survivance, l'image du corps et le signifiant du langage, l'exubérance de la vie et l'exubérance de la mort » (Image survivante 270). Cette notion met en scène des déplacements tant sémiotiques que temporels. On retrouve chez Sigmund Freud une conception du symptôme comprise comme la réalisation de désirs contradictoires (Image survivante 298) où la signification, plutôt que de s'isoler dans l'univoque, se dédouble créant ainsi des situations contradictoires où le sens se déplace, migre sur l'axe diachronique, dans le temps. «Le symptôme — telle est son étymologie — renvoie à ce qui tombe, non à ce qui signifie.

détails qui n'adhèrent pas avec leur époque, où par exemple des figures païennes de l'antiquité grecque ressurgissent dans des scènes chrétiennes de la Renaissance. C'est précisément là qu'il y a un effet de mémoire, où les survivances du temps et de l'histoire surgissent. C'est ici que réside le symptôme. 
Avec lui, les signes eux-mêmes explosent : ils jaillissent en gerbes, puis ils s'écroulent avant un nouveau feu d'artifice» (Image survivante 298). L'étymologie du terme symptôme vient du latin sumptoma qui signifie «coïncidence» ou «accident». Le symptôme arrive de manière impromptue: il jaillit, mais sa signification demeure instable. Lorsque celui-ci apparaît, il ne peut être immédiatement déchiffrable. Il est plutôt un signe qui peut convoquer les traces inconscientes ${ }^{9}$ du passé et les ramener au présent.

\subsection{La mémoire et l'oubli}

Avec les notions de survivance et de symptôme se dégage une sémiotique qui lie la mémoire - inconsciente ou pas — aux restes indiciels. Le fonctionnement de la mémoire, du moins dans sa conception psychanalytique, met en jeu une temporalité qui échappe à la ligne du Chronos. Parler d'anachronisme et d'inexactitude du passé implique une conception du temps qui procède plutôt par montage et qui relève d'un rapport à la mémoire qui est différent. Comme Didi-Huberman le souligne : «la mémoire est psychique dans son processus, anachronique dans ses effets de montage, de reconstruction ou de "décantation" du temps. On ne peut pas accepter la dimension mémorative de l'histoire sans accepter, du même coup, son ancrage dans l'inconscient et sa dimension anachronique» (Devant le Temps 37). Les notions de survivance et de symptôme permettent de lier les traces de la psychè à l'histoire en proposant une vision

\footnotetext{
${ }^{9}$ Dans Les escaliers de Chambord, comme nous le verrons, le personnage principal est confronté à une panoplie de signes et d'objets qui apparaissent sous ce registre, c'est-à-dire comme des signes dont le sens profond ne peut être spontanément déchiffré.
} 
du passé inchoative, systématiquement en mouvement. Le passé est appelé à revenir, mais toujours en questionnant la mémoire et l'oubli, et en proposant un savoir marqué par sa propre indicibilité. Le savoir que nous révèlent les symptômes est inquiet, impuissant : il déstabilise la représentation.

La survivance relève donc à la fois du souvenir et de l'oubli, et doit se saisir comme une marque de l'inaperçu. La survivance se caractérise par cette tension entre l'oubli, le glissement du sens et la retrouvaille inattendue. Dans son ouvrage La mémoire, l'histoire, l'oubli, Paul Ricœur écrit : «Si un souvenir revient, c'est que je l'avais perdu ; mais si malgré tout je le retrouve et je le reconnais, c'est que son image a survécu » (557). Loin d'être une expérience de destruction, l'oubli a une valeur plutôt positive. Chaque souvenir oublié dissimule des traces dont la signification profonde ne peut jamais être immédiatement révélée. Ricœur poursuit : «La solution de la survivance est radicale. Elle consiste en une chaîne de propositions désimpliquées du phénomène de la reconnaissance. Reconnaître un souvenir, c'est le retrouver. Et le retrouver, c'est le présumer principiellement disponible, sinon accessible. Disponible, comme en attente de rappel, mais non à portée de main » (561). C'est ici que nous retrouvons la quête de Pascal Quignard : explorer ce réservoir latent du passé inaccessible — immédiatement — à la conscience. Le Jadis s'érigera sur cette quête d'absences. Ce sont ces traces survivantes, ces déchets symboliques, ces symptômes, qui animent l'ensemble de la quête quignardienne tant dans Dernier royaume que dans Les escaliers de Chambord; car il s'agit bien d'une quête vers un passé immémorial, disponible, mais pas toujours à portée de main. 


\section{CHAPITRE 2}

\section{Le Jadis: Conceptualisation du temps dans Dernier Royaume}

L'anachronisme est donc ce rapport au temps qui est en décalage avec l'acceptation chronologique du temps. Il est un outil conceptuel qui nous permet de déployer une conception du passé ouverte, mouvante, trouble, et toujours en devenir. Il permet de questionner les ramifications conscientes ou inconscientes de la mémoire et de questionner ses zones d'ombre. Pascal Quignard développe dans sa série Dernier Royaume une conception du temps anachronique originale. Tout d'abord, il pense l'anachronisme aussi en opposition à la discipline historiographique. Dans un fragment du troisième tome du Dernier Royaume, Quignard souligne l'importance d'offrir une méthode alternative à la discipline historiographique. Plutôt que de présenter l'histoire comme étant la prérogative des historiens, il développe une figure susceptible d'offrir une vision concurrente de l'histoire. Il écrit : «Il faut défendre les antiquaires et les opposer aux historiens. Il s'agit de mettre en valeur les anecdotiers et la récolte qu'ils font des faits divers pour les opposer au camouflage et à la Propaganda» (Abîmes 37). Contre l'Histoire officielle, celle des grands hommes d'État, l'histoire des vainqueurs comme dirait Walter Benjamin, Quignard propose de creuser le passé et d'en restituer les figures oubliées par l'histoire et de montrer leur contemporanéité. Mais Quignard pousse la notion d'anachronisme beaucoup plus loin et tente de faire remonter un passé vertigineusement lointain. Dans le présent chapitre, nous allons présenter comment Quignard élabore une temporalité radicale que l'on doit penser comme un temps de 
l'origine, celui du Jadis. Mais avant, nous devons examiner la forme fragmentaire que prend la série Dernier Royaume. Ainsi, nous serons en mesure de comprendre la particularité de la méthode — ou de l'absence de méthode — d'écriture de Quignard et comment celle-ci s'avère solidaire du geste « historien » de l'antiquaire.

\subsection{Fragment}

Le lecteur qui entre dans le Dernier Royaume de Quignard bute immédiatement contre une curiosité : celle de la forme. Quignard rejoint une riche tradition d'auteurs qui ont placé l'écriture fragmentaire au centre de leur démarche. Cette exigence du fragmentaire, cette exigence du discontinu, s'avère chez Quignard un geste empreint d'une volonté archéologique visant à déterrer des objets enfouis dans la mémoire. Comme nous l'avons vu, le fonctionnement de la mémoire se déploie essentiellement avec cette tension qu'elle maintient avec l'oubli. Quignard dira que «la mémoire est d'abord une sélection dans ce qui est à oublier, ensuite seulement une rétention de ce qu'on entend mettre à l'écart de l'emprise de l'oubli qui la fonde » (Le Nom sur le bout de la langue 66). La mémoire apparaît comme ce magma informe et fragmentaire distendu avec l'oubli, et où chaque terme — mémoire et oubli — apparaît comme une face complémentaire de la même opération. L’oubli, qui a aussi un potentiel créateur, devient ici structurant de la démarche d'écriture quignardienne. Ce qui doit faire l'objet d'une rétention est précisément ces éléments du temps qui ont été rejetés par l'histoire et le «savoir historien ». L'intérêt du fragment pour Quignard réside alors dans la 
convocation d'un passé fulgurant ${ }^{10}$ et disparate qui refait surface dans le temps présent et qui met à mal toute logique systémique. La méthode d'écriture de Quignard ne fait pas système ; elle échappe au carcan des catégories littéraires.

Avec Régine Robin, nous pouvons relever deux autres figures complémentaires à celle de l'antiquaire qui symbolisent l'inactualité d'une démarche fragmentaire mettant au cœur de ses préoccupations un rapport au temps anachronique. Il s'agit de la figure du chiffonnier et de celle du spectre. La première est empruntée par Walter Benjamin à Charles Baudelaire, c'est-à-dire la figure du chiffonnier du XIX ${ }^{\mathrm{e}}$ siècle chargé de ramasser «tout ce que la grande cité a rejeté, tout ce qu'elle a brisé . . . . Il compulse les archives de la débauche, le capharnaüm des rebuts. Il fait un triage, un choix intelligent; il ramasse comme un avare un trésor, les ordures qui, remâchées par la divinité de l'industrie, deviendront des objets d'utilité ou de jouissance » (Benjamin, Charles Baudelaire 51). Ici, le chiffonnier procède par accumulation de fragments, d'ordures et de déchet oubliés, à un triage, mais aussi un montage des fragments épars qui permettra « de discerner dans des assemblages inédits quelque chose de voix oubliées » (Robin 55). Nous retrouvons un geste analogue chez Quignard alors qu'il ressuscite des figures et des objets oubliés par l'histoire. Tel le chiffonnier, Quignard erre, emporté par une quête ayant pour objet «les chiffons de la parole et les lambeaux du langage»(Zhuo 523).

\footnotetext{
${ }^{10}$ L'image vraie du passé, pour reprendre l'expression de Walter Benjamin, «passe en un éclair. On ne peut retenir le passé que dans une image qui surgit et s'évanouit pour toujours à l'instant même où elle s'offre à la connaissance» ("Thèses sur l'histoire », Euvves III 293). Comme nous le verrons dans Les escaliers de Chambord, les traces du passé resurgissent dans le moment présent, mais leur « lisibilité » n'est jamais en tout temps accessible. Leur interprétation est en différé, et survient au moment ou nous nous y attendons le moins, de manière impromptue.
} 
L'errance est donc alimentée par une quête. Comme Quignard l'écrit dans Sur le jadis : « quand on erre, on ne se dirige pas au hasard. On va où le perdu attire» (18).

La deuxième figure, celle du spectre, du revenant, de la survivance, connote «les retours du refoulé, mais aussi toutes les bifurcations, les voies non empruntées par l'histoire, les vaincus, les solutions abandonnées, les utopies étouffées. Le spectral, ici, est l'espace tiers qui va permettre une part de l'héritage, le passé ouvert dans ce qu'il a encore à nous dire et dans ce que nous avons encore à lui dire » (Robin 56). L'ouverture de l'histoire est marquée par ce qui fut jadis perdu, ce qui manque, ce qui revient, mais toujours sous le signe de l'absence et non de la pleine présence, ne pouvant ainsi garantir aucune vérité stable. Le spectre fragmente le continuum du temps et célèbre la discontinuité. Le fragment devient ici la forme, le langage, permettant de pointer vers cette perte. Le spectre rôde toujours, mais il n'est jamais pleinement présent.

Ainsi devons-nous comprendre le fragment : il est solidaire d'une méthode qui active une réflexion sur la temporalité. Dans Une gêne technique à l'égard du fragment, Quignard apporte la précision suivante : «Les mots latins de fragmen, de fragmentum, viennent de frango : briser, rompre, fracasser, mettre en pièces, en miettes, en poudre, anéantir. En grec, le fragment, c'est le klasma ... le morceau détaché par fracture, l'extrait, quelque chose d'arraché, de tiré violemment» (33). Le fragment, beaucoup plus qu'un simple exercice de style, s'avère être un art de la discontinuité, qui rompt, brise, déconstruit la langue et nos habitudes de lecture, mais qui, dans un mouvement paradoxal, permet de relier plusieurs éléments épars. C'est ainsi que dans Dernier Royaume la forme fragmentaire devient elle-même un appel à ce qui manque, un moyen 
de pointer vers la perte, l'absence et le silence. Comme Martine Motard-Noar en a fait l'observation très juste, la typographie même du Dernier Royaume — ou chaque fragment se voit séparé par des astérisques — marque la volonté de disloquer chacun des fragments et de rompre avec une lecture qui se voudrait linéaire, créant, pour ainsi dire, «une ponctuation de la perte» (Motard-Noar). L'astérisque isole, articule l'espace de lecture imposant une pause, un silence entre chaque fragment, agissant ainsi comme une suspension de la signification : le sens se liquéfie et nous glisse tranquillement entre les doigts. Il y a ainsi une volonté chez Quignard de «laisser du blanc entre les fragments dans le livre », de «ne pas terminer les phrases, les pensées, les liaisons » (Sordidissimes 205).

Le sens entre les fragments et entre les diverses sections maintient une certaine ouverture. Un simple coup d'œil à la table des matières du Dernier Royaume nous donne un indice supplémentaire, alors que nombre des titres donnés aux diverses sections sont placés entre parenthèses ${ }^{11}$, comme si Quignard ne pouvait énoncer avec certitude les thématiques regroupant les fragments. Il crée une épochè, une mise en suspension, une mise entre parenthèses du sens. L'acte d'écriture et de lecture demeure ainsi inachevé, ouvert. Pour Motard-Noar, la lecture des fragments quignardien ne se restreint pas à des liens logiques, successifs, et «chronologiques » à la lecture du texte, mais renvoie à «un contexte bien plus élargi, dans les échos à chercher au niveau du livre [voire de la série] dans sa totalité » (Motard-Noar).

\footnotetext{
${ }^{11}$ Pour illustrer ce fait, dans Sur le jadis, on retrouve par exemple des chapitres intitulés «(Le lac de Garet)», «(Le thé) », "(Le silence à l'état solide)», «(La liste des ombres)», etc. Ces titres entre parenthèses n'apparaissent pas en tête des chapitres mais seulement à la fin, dans la table des matières.
} 
D’un côté Quignard tranche, découpe, déconstruit le sens du discours, mais simultanément, tente de reconstruire, de remonter, un sens perdu. Bénédicte Gorrillot souligne cette tension en ces termes :

«En déconstruisant par fragmentation la langue discursive normative, l'écrivain se donne ainsi les moyens de retrouver un langage d'avant-la culture, un langage du Jadis. Non seulement les coupes (les coups de ciseaux) cassent les codes linguistiques aliénant l'immédiateté de la sensation individuelle. Mais elles permettent aussi de figurer la réception spontanée, désordonnée, que nous avons eue du monde » (Gorrillot 260).

Par le geste de coupure, Quignard ne se livre donc pas strictement à une fragmentation du discours qui risquerait d'échouer à rejoindre une totalité. Il n'y a pas, comme chez Maurice Blanchot, par exemple, d'absolu du fragmentaire où la forme — ou l'absence de forme - échouerait à rejoindre le tout et ne parviendrait qu'à le signifier «en le suspendant, voire en le brisant» (Blanchot 518). Chez Quignard, «les fragments sont bien des morceaux de textes séparés, discontinus, ils sont également un ensemble d'éléments organisés, à l'intérieur duquel des fragments sont en série, se répondent, se font écho ou se font face, sont liés » (Rodrigues 125). Les fragments sont disparates mais toujours reliés entre eux. Quignard re-lit des figures intellectuelles oubliées dans le passé, mais il procède aussi à une liaison de reliques (Gorrillot 261), c'est-à-dire de reliquiae, de restes — matériels — qui cherchent à indiquer un passé lointain. Quignard donne alors au fragment, et par extension à son geste d'écriture, une fonction qui est bien particulière, soit celle de relier entre eux des fragments du passé, et surtout, de les relier à une temporalité originaire qui fut jadis unitaire. Les fragments signalent un souvenir ancien, 
absent, perdu, oublié et indicible. Ces souvenirs sont ceux appartenant à ce que Quignard nomme le Jadis.

\subsection{L'origine}

Jusqu'à présent, nous avons principalement examiné la forme particulière qu'arbore le Dernier Royaume. La forme rejoint dans une symbiose le contenu de l'œuvre de Quignard. La dimension indicative des fragments-signes contribue à la conception originale et originaire du temps, radicalement inorienté et déchronologisant, qui remet en question la tripartition classique du temps entre le présent, le passé et le futur. Dans un fragment des Petits traités (1990), Quignard pose les premiers jalons d'une conception du temps libéré de la continuité et de la succession :

Je compte les dimensions qui font le temps et il me semble aussitôt qu'il n'y a pas lieu de n'épouser qu'une seule. On racontait autrefois que l'introduction de la langue dans le monde avait elle-même introduit deux continents jusque là inconcevables: d'une part quelque chose d'extraordinairement désynchronisé en regard du réel et qu'on a appelé depuis la mémoire; d'autre part l'anachronie à l'état pur — qu'on appelle aussi l'imaginaire. (490)

D'une part, Quignard assigne à la mémoire ce privilège d'ouvrir un monde inconcevable libéré de la synchronie et d'autre part, il assigne à l'imaginaire un rapport au temps qualifié de purement anachronique. La conception du temps qui ressort de ces lignes souligne l'opposition directe entre mémoire et fiction, où le réel demeure inaccessible. Dans Sur le jadis, Quignard reviendra sur cette facette de l'anachronie, mais cette fois-ci, en disant que «[1]e pur jadis est anachronie pure (comme l'image est absente de l'objet)» (152). 
Le Dernier Royaume est le lieu où se déploiera la définition du Jadis et où Quignard élaborera une définition ouverte qui se creuse, se précise au fil de la lecture et du déploiement des fragments. Ce programme apparaît dès les premières pages du premier tome intitulé Les ombres errantes. Quignard écrit : «La scène où toute scène prend origine dans l'invisible sans langage est une actualité sans cesse active » (15). Cette scène originaire semble hanter toute la démarche quignardienne. Le Jadis est une temporalité qui tente de remonter vers l'origine de l'homme, l'origine du monde, et de faire resurgir des «vestiges d'une scène antérieure que nous ne connaîtrons jamais » (Quignard «Dialogue avec Tiphaine Samoyault », 202), c'est-à-dire d'une scène qui gît au fond de notre imaginaire. Scène impossible à saisir, elle demeure néanmoins structurante, inconsciemment déterminante, hantant, tel un fantôme, nos rêves, notre imaginaire et notre présent. «Nous sommes à la merci d'images qui n'ont aucune source visuelle en nous. Nous avons vécu avant de naître»(28), écrit Quignard dans les premières pages de Sur le jadis.

Nous sommes à la merci d'une origine impossible à voir (car après tout, personne ne pourrait accéder à la scène de sa propre conception) — c'est-à-dire de cette scène originelle qui a permis notre apparition dans le monde -, mais qui laisse des traces et qui oriente notre vision. C'est ainsi que l'ensemble de Sur le jadis sera ponctué par ce souci de ce que Quignard nomme le monde vivipare, intra-utérin, le monde d'avant la naissance, un monde pur, d'avant le langage, le monde présymbolique de l'enfant qui n'a pas encore acquis le langage, c'est-à-dire celui de l'infans (49). Quignard écrit :

La porte pour entrer dans le monde n'est pas la naissance. Elle n'est pas le froid ni la lumière. Elle n'est pas le corps distinct, sexuel, abandonné, 
soufflant, expulsant, manquant, parlant. Ni la faim, ni la carence, ni le besoin, ni le groupe, ni la langue naturelle, ni la conscience qui fait revenir la voix du groupe en écho.

La porte est l'origine. L'origine eut lieu dans un monde interne relié encore à la jouissance animale elle-même.

Je parle de la jouissance sexuelle, originaire, aoristique, inorientée, antémorphique. (Sur le jadis 60)

L'origine est donc une conception de la temporalité qui échappe à toute tentative de réappropriation consciente et qui ne serait pas susceptible de faire l'objet d'un savoir «historien ». Elle est une temporalité inorientée, antémorphique, c'est-à-dire antérieure à la matière, antérieure à l'apparition dans le monde, et dont il est impossible de retracer précisément le début et la fin. Ce monde de l'animalité ${ }^{12}$ est un espace autre, un «site originaire qui n'est pas de ce monde » (Les Paradisiaques, 114).

Il y a donc un effet de survivance, un resurgissement inopiné et imprévisible d'une mémoire si lointaine qu'elle échappe au principe de datation. Pour Quignard, cette scène originaire « dont tous résultent, n'est jamais passée. Elle constitue le jadis éternel et cet éternel est négatif .... La scène dont nous sommes la suite nous escorte sans qu'elle n'apparaisse jamais » (Sur le jadis 200). Cette scène précède alors toute visibilité, elle demeure systématiquement cachée, comme une figure ou une image qui agit dans l'ombre. Pour comprendre la catégorie d'origine, il ne faut donc pas la penser comme une genèse, une explication causale qui permettrait de mettre à nu le passé. L'origine comme

\footnotetext{
${ }^{12}$ La question d'un fond «animal» traverse l'ensemble des Petits traités et du Dernier Royaume et pose nombre de réflexions d'ordre anthropologique, ontogénétique et phylogénétique. Ceci rejoint aussi des considérations sur l'acte d'écriture et de lecture. Jean-Louis Pautrot écrit, en commentant un passage des Petits traités : «La littérature, selon le traité quignardien, suppose donc un ancrage paradoxal, affectif, animal, de l'écriture qui se devine dans le non-dit, dans la trace d'une présence brute, qui s'éprouve en supplément connotatif et allusif de l'écrit » 48). La littérature devient un acte de prédation, de recherche d'un fond «brute », et cherche à abolir, ou du moins à questionner « la frontière incertaine entre l'humain et l'animal» (Alvarez 232).
} 
catégorie, pour reprendre les mots de Walter Benjamin, «ne désigne pas le devenir de ce qui est né, mais bien ce qui est en train de nâtre dans le devenir et le déclin. . . . Elle demande à être reconnue d'une part comme une restauration, une restitution, d'autre part comme quelque chose qui est par là même inachevé, toujours ouvert » (Origine du drame baroque 43). L'origine et le Jadis se construisent donc en opposition avec la notion de passé : ils ne sont pas finis, mais en constant devenir. Leur registre temporel appartient à l'anachronie.

\subsection{Le Jadis}

Le Jadis est cette temporalité propre à l'origine et il permet de déployer un temps qui se situe hors chronos. Il se veut inachevé, inorienté, ouvert. Il n'est pas un temps du passé, mais se construit plutôt en opposition au temps passé :

Le jadis par rapport au passé a pour premier trait de ne pas avoir nécessairement été. Le jadis ne figure ni au nombre des étants ni au nombre des ayant été, car il n'a pas encore fini de surgir. Le jadis est un puits plus vaste que tout le passé .... Il faut distinguer jadis immémorial et passé ayant été présent de même que la source est une image qui manque, il y a un passé qui manque (Sur le jadis 149-150).

Cette définition que nous offre Quignard s'avère excessivement ouverte sur le plan ontologique. Autrement dit, on est hors ontologie, on ne peut pas assigner au Jadis le statut de chose, ou d'objet stable ayant existé. Il n'est pas comme ce savoir historique sur lequel nous pourrions nous pencher; que nous pourrions analyser, interpréter et soumettre à une réinterprétation. Ici, l'interprétation historienne est impossible et inaccessible. Par l'écriture, par la narration — historienne, romanesque ou poétique «[o]n change de passé alors qu'on ne change pas de Jadis » (Sur le jadis 17). Le Jadis est 
éternel, impossible à lire ou relire, car le Jadis n'est pas un «ça-a-été », pour reprendre l'expression de Roland Barthes. ${ }^{13}$ Sa lisibilité n'est jamais immédiatement accessible, d'où la nécessité d'une médiation (signe, fragment, symptôme, indice, rebut) pour faire resurgir le Jadis. Il n'a pas nécessairement été, il n'est pas une action close que l'on pourrait conjuguer au passé composé, ou à l'imparfait. C'est un passé qui manque, car il n'a pas fini de surgir. C'est un temps aoriste.

Ce terme d'aoriste est un concept clef qui traverse l'ensemble du Dernier Royaume et brouille les catégories de passé, présent et futur. Le geste énonciateur de l'aoriste est « déchronologisant $» .{ }^{14}$ L'aoriste est un temps verbal que l'on retrouve entre autres dans le grec ancien et qui exprime «un passé indéterminé, car il est impossible de définir exactement le moment dans le passé par rapport auquel est localisé le procès ; un fait pur et simple ou une vérité d'ordre général» (Messager 206). C'est un temps de l'origine qui ne peut être situé avec précision sur la ligne du temps, et qui ne trouverait pas d'équivalence dans le français moderne. Si sa spécificité échappe à la langue française, on retrouve tout de même certaines tentatives de palier à cette catégorie manquante de notre système verbal en assignant au passé simple une dimension aoristique. Dans Sur le jadis, Quignard consacre un chapitre à cette notion. Il écrit :

Pourquoi suis-je un homme qui écrit toujours au passé simple? Parce que j'écris au singulier....

L'aoriste est une mélancolie qui monte aussi lentement que la neige qui tombe et qui transforme tout ce qu'elle touche.

L'aoriste n'enseigne pas.

C'est une irruption du perdu elle-même perdue et à partir de laquelle le perdu n'est pas préservé. Car ce qui définit le perdu est sans retour. (123)

\footnotetext{
${ }^{13}$ Voir Barthes, Roland. «La chambre claire. Note sur la photographie», dans Euvres complètes V, Paris, Seuil, 2002.

${ }^{14}$ Voir notre premier chapitre p. 13.
} 
L'aoriste, n'est donc pas une mélancolie au sens psychanalytique du terme ${ }^{15}$. Il pose la question du deuil du temps, mais il présente aussi un aspect imperfectif parce qu'il est toujours en train de se faire. La formulation choisie par Quignard évoque un double mouvement. Une mélancolie, un passé endeuillé et perdu qui monte, ou qui remonte le temps, et qui tombe comme la neige, comme un signe qui s'écroule et qui échappe à une signification sûre et certaine. Quignard trouve donc une forme d'énonciation qui fait remonter la perte, qui fait irruption, sans pour autant être capable d'asseoir un savoir du passé stable. Par la suite, Quignard creuse le concept d'aoriste en lui assignant une «amplitude sémantique» qui en «métamorphose des acceptations premières strictement linguistiques, grammaticales - et [les convertit] en quelques figurations plurielles d'un temps sans fond, illimité » (Messager 209). Quignard creuse ce concept avec des images et en le distinguant d'autres temps verbaux. Il n'est pas le passé composé (ça-a-été), mais un temps qui fonce, qui transforme la matière comme la neige tombante qui touche le sol, qui émerge dans le présent, et qui ne présente pas de limites. «Le mot ancien a-oristos nomme en langue grecque ce sans limites que la limite (l'horizon temporel) projette en amont de lui-même. "L'aoriste" est cet "a-horizon". . . . C'est le

\footnotetext{
${ }^{15}$ Dans son essai Deuil et mélancolie, Freud définit la mélancolie comme étant « un point de vue psychique [se caractérisant] par une dépression profondément douloureuse, une suspension de l'intérêt pour le monde extérieur, la perte de la capacité d'aimer, l'inhibition de toute activité et la diminution du sentiment d'estime de soi » (146-147). De façon analogue au processus de deuil, qui se caractérise par une réaction de la libido à la perte de l'objet aimé, la mélancolie apparaît comme la perte d'un objet d'amour, « à laquelle cependant ne fait pas suite, contrairement à ce que l'on pourrait attendre, un transfert de la libido sur un nouvel objet, mais son retrait dans le moi, narcissiquement identifié à l'objet perdu » (Agamben, Stanze 47), processus qui, contrairement au deuil, se fait hors du champ de la conscience. Voir Freud, Sigmund. « Deuil et mélancolie », dans Métapsychologie, Trad. Jean Laplanche et B. Pontalis, Paris, Gallimard, Folio, 1968, et Agamben, Giorgio. Stanze. Parole et fantasme dans la culture occidentale. Trad. Yves Hersant. Paris : Rivages poche, Petite Bibliothèque. 1998.
} 
fond de ce passé dépourvu de frontière» (Sur le jadis 205). Il ne s'agit donc pas d'un «ça-a-été », ou d'un «ce fut », mais bien d'une revenance dans le présent : «Ce fut est là » (Sur le jadis 43). Le Jadis s'ouvre sur le présent et au-delà.

Le Jadis, n'est donc pas un passé — un passé composé où l'action serait prisonnière de sa propre finitude —, mais un «ce fut » actualisé et toujours en devenir, c'est-à-dire «un passé refondu en liquide » (Sur le jadis 158), un passé désolidifié et donc infiniment malléable. Il est un présent intense et absolu, le «présent du présent » (Sur le jadis 159). Ce n'est pas une vision passéiste du temps passé, futuriste du futur, ou présentiste du présent, mais un horizon, «un espace de réflexion et de création hors de la modernité»(Ruffel 106). D'où la dimension intrinsèquement contemporaine que la notion de Jadis revêt: c'est une fracture dans le temps, un déphasage, un pur anachronisme. Le Jadis rejoint ce que Giorgio Agamben entendait par la notion de « contemporain »:

La contemporanéité s'inscrit, en fait, dans le présent en le signalant avant tout comme archaïque, et seul celui qui perçoit dans les choses les plus modernes et les plus récentes les indices ou la signature de l'archaïsme peut être un contemporain. Archaïque signifie proche de l'arkè, c'est-àdire de l'origine. Mais l'origine n'est pas seulement située dans un passé chronologique : elle est contemporaine du devenir historique et ne cesse pas d'agir à travers lui, tout comme l'embryon continue de vivre dans les tissus de l'organisme parvenu à maturité, et l'enfant dans la vie psychique de l'adulte (Agamben 28).

D'une part, la notion de contemporanéité, tout comme le Jadis, pose cette dette de sens. Le présent est tributaire d'un fond lointain et archaïque. D'autre part, l'origine se situe dans le devenir historique. L'analyse de Simon Saint-Onge dans son article «Le temps contemporain ou le jadis chez Pascal Quignard» synthétise la position quignardienne 
exactement en ces mêmes termes : un éclatement de la chronologie qui place l'extase au centre de ses processus de signification. Saint-Onge écrit : «le Jadis se délivre dans un processus de figuration extatique . . c c'est-à-dire qu'il s'agit d'un faire créatif » (171), une force toujours à venir. Le temps pour Quignard est un pur issir (Sur le jadis 32), une porte de sortie vers une extase incessante.

\subsection{La perte}

Le Jadis est une temporalité particulière où des vestiges archaïques sont appelés à faire irruption dans le temps présent et à en déchirer la représentation. Le Jadis resurgit d'un passé lointain, émerge des eaux profondes de la mémoire, venant interrompre cette représentation factice du temps compris comme le temps de la chronologie qui se déploie suivant un ordre logique d'apparition de moments hétérogènes. Le Jadis défait les chronologies, change les rythmes, et nous force à concevoir ce qui nous est contemporain dans sa relation avec son origine, avec ce fond toujours inaccessible submergé sous les flots du temps. La réapparition, la revenance, la survivance, la répétition d'un fond lointain deviennent alors les marques du Jadis et le différencient du simple passé. Dans le troisième tome du Dernier Royaume, Quignard énonce cette précision : «La compulsion de répétition est la force du jadis » (Abîmes 78). Quignard construit alors cette opposition entre passé et Jadis autour de la figure de la répétition et de la perte.

D'un côté, le Jadis est une revenance, une force inépuisable qui ne cesse de survenir, et de l'autre, le passé est marqué par l'expérience de la perte. Quignard écrit : 
Le jeu par lequel un objet tombe puis est repêché. Le jeu fait alterner de façon active absent présent; caché vu; perdu retrouvé ; cette polarisation entre invisible et visible structure le temps binaire. Le premier rythme est celui de la disparition suivie d'apparition. La nativité après la vie sombre, l'après coup suivi du premier monde, tel est le battement de l'originaire. Le temps fort est la perte. Le temps faible le resurgissement. La réapparition n'est qu'un répété. La pulsio n'est que la deuxième fois.

Seul le temps fort, la perte, la naissance font connaître la première fois. (Abîme 85)

Cette dynamique entre deux rythmes offre une dimension matérielle dans laquelle nous pouvons désigner le Jadis. Nous sommes comme ce jeune enfant de 18 mois — cet infans — que Sigmund Freud décrit dans Au-delà du principe de plaisir et qui rejoue inlassablement la scène de la disparition de sa mère. Il tient entre ses mains une petite bobine de bois à laquelle une ficelle est rattachée. C'est un objet de vision qu'il peut manipuler sans en comprendre toutes les significations et les ramifications. Dans un jeu de la perte et des retrouvailles, l'enfant lance au loin cette petite bobine qui disparait sous un rideau. La perte provoque un mouvement ; c'est le moment fort, le moment de convocation du lointain — Fort qui signifie en allemand «loin» — où l'enfant s'écrie «o-o-o-o» en constatant la disparition. Le moment de réapparition, lorsque celui-ci tire sur la ficelle, sera à son tour ponctué «par un joyeux "Da !" ("Voilà !") » (Freud, Au-delà 52). L'idée qui se cache derrière cette anecdote est que le moment de la disparition et de la perte révèle la dynamique particulière. Pour Freud, la disparition de la bobine renvoie à la disparition de la figure maternelle rendue indisponible au regard du jeune enfant. $\mathrm{Ce}$ n'est que par la disparition et la réapparition de la bobine que l'on peut saisir cette autre absence davantage originaire, c'est-à-dire celle de la mère. La bobine, et le jeu dont elle est l'objet, apparaît alors comme un signe pointant vers une absence encore plus profonde 
et lointaine, soit celle d'une disparition indicible. Mais ce réapparaître suscite une certaine joie. Dans la répétition, il y a une certaine volupté, voire un certain sens du sublime. L'absence, tout comme la thématique de l'oubli que nous avons esquissée dans notre premier chapitre, a donc un versant positif.

De façon analogue, nous pouvons avancer que les objets du passé, qui font irruption dans le présent en venant déchirer la trame temporelle, exposent des régions inaccessibles du temps. Comme Didi-Huberman le remarque, la bobine contient en ellemême un pouvoir particulier qui est celui d'être capable de «dévier, disparaître, passer sous un meuble inatteignable, parce que son fil peut casser ou résister . . . et passer ici dans l'inexistence totale. Elle est fragile, elle est quasi. En un sens, elle est sublime » ( $\mathrm{Ce}$ que nous voyons 55). Cette fragilité se conçoit aussi comme une fragilité de la signification. La bobine acquiert une signification fulgurante, qui passe, indique une perte. Mais cette indication n'est que passagère et le signe n'existe «que sur fond de ruine» (Ce que nous voyons 56). C'est ainsi que le Jadis devra resurgir. Il ne sera rendu accessible que partiellement et par la médiation d'objets qui ont pour caractéristique d'être instable sur le plan de la signification, c'est-à-dire des rebuts. Précisément, cette question du rebut sera au cœur du roman Les Escaliers de Chambord. 


\section{CHAPITRE 3}

\section{Les rebuts et les Sordidissimes dans Les Escaliers de Chambord}

Les fonctions déchronologisantes de la temporalité du Jadis sont solidaires d'une méthode qui place le fragment au centre de l'écriture de Pascal Quignard. Si jusqu'à présent nous avons trouvé en le Jadis une temporalité originaire et ouverte, essentiellement anachronique, il importe maintenant de développer une «méthode » qui nous permettra de nous approcher de cette temporalité surdéterminante, toujours en devenir, mais pourtant inaccessible. Dans le roman Les escaliers de Chambord, plusieurs éléments nous permettent de «rejoindre» le Jadis. Tout d'abord, nous voyons dans ce roman une figure susceptible d'incarner ce travail de fragmentation du temps. À travers le personnage d'Édouard Furfooz et la thématique de la collection, nous amorcerons avec Quignard une réflexion sur la notion d'objet et d'absence. Dans un deuxième temps, au travers de la thématique de la collection, nous mènerons une réflexion plus large sur le monde des objets et ainsi, par l'entremise de la figure du déchet, nous dégagerons une catégorie de signes apte à pointer vers le Jadis. Le déchet, pour le dire comme Éric Méchoulan, «est ce qui a déchu de sa fonction d'usage, donc de sa valeur d'échange, il est aussi "ce qui a fait son temps" » (Méchoulan 184). Il a fait son temps, il est vidé de sa temporalité, et attend d'être retrouvé et investi d'une autre signification. Troisièmement, cette chasse aux rebuts nous permettra d'aborder directement la question de la mémoire conceptualisée dans Les escaliers de Chambord. La valeur indicative du rebut sera susceptible de pointer vers des souvenirs refoulés par le personnage de Furfooz. 
Finalement, nous conclurons ce chapitre sur la thématique de l'enfance qui traverse l'ensemble du roman et qui relie les différentes quêtes menées par Furfooz.

\subsection{Le collectionneur}

La figure de l'antiquaire que présente Quignard dans le Dernier royaume nous a permis d'anticiper un «personnage » qui développe un discours en opposition à celui de l'historien — qui serait le gardien d'un discours sur la vérité de l'histoire —, mais qui en plus, préconise une méthodologie singulière. Le geste quignardien de montage de fragments que l'on retrouve dans la figure de l'antiquaire était déjà présent dans le Dernier Royaume: accumulation de fragments, de morceaux épars du passé, de restitution des choses oubliées. Cette figure, à laquelle nous avons joint celle du chiffonnier, occupe une place centrale dans Les escaliers de Chambord alors que l'intrigue du roman se déploie autour du personnage d'Édouard Furfooz, un antiquaire et collectionneur de jouets d'enfants antiques. Personnage excentrique, issu d'une riche famille noble d'Anvers, Furfooz est mû par une insatiable quête d'objets qui l'emporte aux quatre coins de l'Europe et de l'autre côté de l'Atlantique, non sans une certaine frénésie. Cette quête d'objets fait de lui un personnage ex-centrique, sans port d'attache, déraciné, qui est incapable de trouver la quiétude. Quignard écrit : «Il avait beau se déplacer, sans cesse, jour après jour, il n'abordait nulle part. Où était-il ? » (Les escaliers de Chambord 22).

La collection est le motif qui met en mouvement la quête de Furfooz. Le monde des collectionneurs est méticuleusement décrit comme un monde peuplé par « des êtres 
anachroniques» (Lapeyre-Desmaison, Mémoires de l'origine 119) qui échappent au temps linéaire. Quignard qualifie les collectionneurs en ces termes :

Des Pillards, ou plutôt des êtres qui triaient et mettaient en rang d'oignon des vieux trésors, tels étaient les collectionneurs. C'était des pirates éliminés qui soustrayaient des choses à l'usage. C'étaient tout à coup des clés sans portes, des jupes sans corps, des épées sans cadavres, des montres arrêtées. C'étaient des monnaies qui n'achetaient plus rien dans l'univers. C'étaient des jouets sans enfants. ... Et il songeait que plus ces objets pillés et transférés de site en site avaient été gâtés par le passage du temps, moins on les restaurait. Et c'était comme son cœur. Et moins ils avaient d'usage, plus leur valeur marchande s'accroissait. Tout cela était frappé au coin de la démence. (22)

Dans cette description du métier de collectionneur, Quignard attire notre attention sur divers éléments significatifs concernant cet «art» de l'accumulation. Dans un premier temps, le collectionneur profane l'usage originel des objets. La valeur d'usage assignée aux objets devient caduque et se veut même inversement proportionnelle à la valeur d'échange. Plus un objet est débarrassé de son utilité et de sa fonction, plus celui-ci, pour le regard affûté du collectionneur, prend une valeur d'échange. Cet aspect irrationnel, frappé par le sceau de la démence comme le dit Quignard, est une caractéristique importante. Comme le remarquait Walter Benjamin dans son ouvrage consacré aux passages parisiens - haut lieu de la marchandise au XIX ${ }^{\mathrm{e}}$ siècle —, l'important «dans l'art de collectionner, c'est que l'objet soit détaché de toutes ses fonctions primitives » (Benjamin, Paris, capitale du XIX $X^{e}$ siècle 221-222), et que ce détachement permet de redonner une «magie », une marque irrationnelle et fétichiste à la marchandise. L'objetnaufragé, une fois secouru par le collectionneur, acquiert une autre signification qui échappe au regard des non-initiés (Benjamin, Paris, capitale du XIX siècle 224). L'objet se voit investi d'une dimension sacrée, religieuse. Quignard écrit : 
«Tous les collectionneurs qu'il connaissait étaient des jaloux malades. Toute leur convoitise se résumait dans ce désir d'extraire l'objet inestimable du circuit économique et du regard même de ceux qui auraient joui de le voir. ... Par là chacun devenait soi-même un prêtre, un roi assis sur un trésor. Plus encore, on devenait une part de la chair de Dieu, on était vraiment en contact avec l'objet sacré, avec la relique » (Escaliers 185).

L'étymologie du terme «religion» nous le rappelle, le terme religare signifie relier, et l'acte de collection en est un qui relie le disparate, c'est-à-dire qui relie et crée des liens entre des objets qui a priori ne semblent avoir aucun dénominateur commun sur le plan de la signification. Tout au long du roman, c'est ce geste que tente d'accomplir Furfooz : relier des éléments dont la signification ne cesse de lui glisser entre les doigts. Le personnage de Furfooz apparaît donc dans le roman de Quignard comme une figure susceptible de lier des temporalités et des moments disjoints, de lier le monde de l'enfance à celui des adultes. Furfooz crée «une liaison entre les âmes des tout petits enfants qui hurlent et celle des hommes dont la crainte de la mort et le silence ont déjà commencé de figer les traits » (Escaliers 13).

Dans un deuxième temps, au travers du collectionneur, Quignard introduit l'idée de la perte, motif présent dans l'ensemble du roman. Les objets que ramassent les collectionneurs sont des objets, ou des jouets qui ont perdu leurs enfants. Le collectionneur est à la recherche de «tout ce que des petites mains d'enfants devenus des cadavres avaient tenu ${ }^{16} \gg$ (Escaliers 23). Les objets sans usage sont des objets perdus par

\footnotetext{
${ }^{16}$ Déjà, dans cette citation qui se retrouve en début de roman, nous pouvons voir une anticipation du dénouement du roman. Claudette Oriol-Boyer souligne que le motif de la «main » réapparaît à plusieurs reprises tout au long du roman, comme un indice et un rappel tactile de la personne disparue. Oriol-Boye souligne, en parlant de l'image de la main, que celle-ci «peut aussi évoquer des images tragiques : Édouard est d'Anvers (qui signifie main coupée) et il vit avec le sentiment permanent d'une amputation. L'explication apparaîtra à la fin quand on découvrira qu'il fallut extirper de son poing serré des bouts de cheveux noirs et une barrette bleue qui faisaient corps avec sa main. En les lui arrachant, c'est une partie de
} 
leurs propriétaires : ils sont révélateurs d'une absence ${ }^{17}$. Et finalement, le collectionneur est un chasseur, un prédateur, un pirate : il cherche «le butin du passé » et « les trésors du temps » (Escaliers 23). L’objet dépossédé de sa valeur d'usage et de son propriétaire est aussi un objet qui porte en lui une marque de temporalité : il appartient au temps sous la marque de l'origine. Pour Benjamin, «la collection est un phénomène originaire. . . . Le collectionneur, pour qui les choses s'enrichissent avec la connaissance qu'il a de leur genèse et de leur durée dans l'histoire, parvient ainsi à avoir avec elles un rapport semblable qui paraît aujourd'hui archaïque » (Paris, capitale du XIX $X^{e}$ siècle 228). Entrer en contact avec ces objets permet d'en remonter l'origine, d'en mesurer la durée, mais surtout, de les détacher de leurs contextes, leur garantissant ainsi une signification ouverte, perpétuellement en mouvement. Les objets, sous le regard du collectionneur, se trouvent débarrassés de leur âge (Escaliers 11). Dans le désordre fragmentaire que nous a légué l'histoire, le collectionneur profite de l'occasion pour relier les choses éparpillées dans le monde, et engage un combat contre la dispersion.

Mais Furfooz est aussi spécialisé dans la collection de jouets. Le jouet, pour reprendre les mots de Soizic Bonvarlet, «est l'objet inutile par excellence» (4). L'inutilité acquiert une certaine force temporelle. Le jouet apparaît comme une copie d'un modèle, mais ajustée à la main de l'enfant. La petite voiture de jouet miniaturisée ne possède bien évidemment pas la même fonction que la voiture réelle. Elle est réinvestie d'une toute autre fonction. Comme l'écrit Giorgio Agamben, «[1]e pays des jouets est un

lui-même qu'on lui a arrachée.» Voir «Les escaliers de Chambord de Pascal Quignard. Roman d'apprentissage de la lecture et de l'écriture ». Pascal Quignard, figures d'un lettré, Actes du Colloque de Cerisy-la-Salle (août 2004), Paris : Galilée, 2005, p. 160-161.

${ }^{17}$ Nous y reviendrons plus loin dans ce chapitre. 
pays dont les habitants célèbrent des rites, ou manipulent des objets et des formules sacrées, dont ils ont pourtant oublié le sens et la fonction» (129). Le jouet demeure manipulable, mais sa signification intrinsèque demeure instable. Comme la bobine avec laquelle s'amusait le jeune enfant que nous décrivait Freud : dans le cadre du jeu, celle-ci acquiert une nouvelle fonction, perdant ainsi son sens premier. Une fois le jeu terminé, le jouet est rangé, perdant simultanément sa signification. La marque temporelle du jouet se situe donc sur l'axe diachronique : elle survient, s'estompe, disparait, survit, réapparaît.

\subsection{Le rebut}

Le collectionneur accumule donc des objets du passé à la signification instable. Dans Les escaliers de Chambord, la valeur sémiotique des jouets collectionnés dépasse le simple acte de possession : Furfooz traque des objets, et ceux-ci acquièrent un statut qui implique des déplacements sur l'axe diachronique, des retours en amont et une structure anachronique. Autrement dit, cette quête de jouets, qui emporte Furfooz tout au long du roman, en dissimule une autre. L'objet dépouillé de ses fonctions, en réalité, permet de retrouver une temporalité beaucoup plus fondamentale et originaire. Quignard écrit : «Les jouets, ce ne sont pas les objets de ce monde. Il y a eu un autre monde qui a précédé cette lumière où nous baignons. [...] Il se répétait avec conviction : il y a deux sortes d'objets dans le monde. Les objets d'ici-bas et les objets d'au-delà. Les objets qui appartiennent à l'usage et les objets qui appartiennent au sans usage » (Escaliers 39). Les jouets ont donc ce statut privilégié de pointer vers un autre monde, un autre temps 
inaccessible. C'est précisément l'absence de fonction qui autorise le signifiant à glisser tranquillement vers un autre sens perdu.

Quignard développera — au-delà du jouet — une riche réflexion autour de la notion d'objet. Un des moments les plus marquants dans Les escaliers de Chambord survient lorsque Furfooz se retrouve en Italie afin d'y conduire des affaires pour l'une de ses boutiques qui se trouvent dans ce pays. Alors qu'il déambule sur une plage donnant sur la Méditerranée, il fait la découverte parmi un amas de débris d'une petite barrette bleue en plastique représentant une grenouille. Cette découverte suscitera un fort émoi inexplicable : «Le désir impulsif de la prendre, la jubilation qu'il avait ressentie à la découvrir, à l'isoler par le regard parmi les déchets, parmi les plumes de mouettes, les bidons d'huile, les excréments, les ceps et les pelures de fruits, à se pencher sur elle et à s'en saisir lui étaient incompréhensibles »(Escaliers 39-40). Il s'agit de la première occurrence de cet objet dans le roman. Pour une raison qui lui échappe, Furfooz conservera précieusement cette relique, ce déchet, au fond de sa poche. La barrette interviendra à plusieurs moments clefs dans le roman et sera le déclencheur de visions et de souvenirs instables et évanescents ${ }^{18}$. Le rebut a donc une fonction indicative susceptible de ramener brusquement des souvenirs, mais dont la signification ne pourra être reconstruite dans sa totalité. La signification apparaît, mais à cette étape, elle n'est «ressentie » que sur le mode de l'affect. Quignard écrit : «Le mouvement désordonné de

\footnotetext{
${ }^{18}$ Tout au long du roman, on dénombre environ une quinzaine d'occurrences et de références à cet objet. La petite barrette flotte entre les pages du roman et sert en quelque sorte « de fil d'Ariane pour le guider dans le labyrinthe de sa mémoire » (Demanze 122).
} 
la barrette bleue était celui de la douleur » (81), mais une douleur qui demeurera indicible jusqu'à la toute fin du roman.

L'importance de la thématique du déchet n'est donc pas fortuite et fait aussi l'objet d'une thématisation toute particulière dans le Dernier royaume. Le quatrième tome de la série intitulé Sordidissimes, comme le titre le laisse entendre, est consacré au rôle et à la fonction des objets sordides. Comme Quignard le mentionne : «Ce livre que j'écris et que je nomme Sordidissimes je l'ai déjà écrit, jadis, sous forme de roman. [...] Ce sont Les escaliers de Chambord» (Sordidissimes 77). On retrouve en effet dans ce volume une réflexion similaire sur le statut des déchets et leur habileté à entrer dans des réseaux de signification, ou plutôt à déplacer les significations. La filiation du terme sordidissime remonte à Caius Albucius Silus, figure à laquelle Quignard a consacré un roman. La thématique de l'objet et du sordide apparait dès lors comme un phénomène hétérogène dans le temps, aux manifestations multiples, et dont l'essence réside dans l'exhibition d'une passion «de l'objet qui n'est pas de ce monde ( (Sordidissimes 54). Quignard écrit :

Ce que le rhéteur romain Caius Albucius Silus appela au Ier siècle avant Jésus-Christ sordidissima, ce que Georges Bataille, à Paris, à la fin des années cinquante appela "part maudite", ce que Jacques Lacan, à Paris, au début des années soixante, appela "objet petit a", ce que les New-Yorkais à la fin du XXe siècle appelèrent junk, jadis sur les parois des corps, c'étaient des traces silencieuses, des lignes, des tatouages, des peintures, des parures. Jadis, au Japon, ceux qui étaient «blessés» par l'amour cherchaient à exhiber des marques de «blessures» sur le corps. (Sordidissimes 54).

Bien que cette citation incorpore nombre de réflexions qui excèdent le cadre de notre analyse, il est important de souligner sa portée théorique. Premièrement, la réflexion de 
Quignard sur l'objet tente de traiter de l'abject, de la saleté, et de lier ces thématiques à la question du temps originaire. Dans Sordidissimes, Quignard reprend les mots de Montaigne «Le sperme est une saleté sordide quel qu'en soit le destin. Montaigne : Quel monstre est-ce que cette goutte de semence de quoy nous sommes produits » (46). Notre origine, cette scène irreprésentable et inaccessible dont nous sommes tous issus, est imprégnée du sordide. L'abject oscille entre, d'un côté, la passion hors de ce monde, et de l'autre, le dégoût, la surprise, ce qui perturbe, révulse, et dégoûte. Même si la scène est inaccessible, il en persiste néanmoins des traces et des résidus.

Les sordidissimes, les objets-déchets, sont alors des marques survivantes d'un passé, mais aussi des symptômes qui font irruption dans le monde phénoménal et qui en perturbe le sens. Ils sont des symboles en mouvement et dont l'identité première est rendue caduque. La petite barrette bleue n'est pas intéressante en soi : nous avons oublié que celle-ci avait pour fonction de retenir les cheveux d'un enfant. La valeur iconique de la grenouille ne revêt d'ailleurs pas plus d'importance : ce qui importe c'est sa valeur de symptôme et sa capacité à renvoyer à un autre signe. Le déchet est alors beaucoup plus proche de ce que Charles Sanders Peirce appelait un indice, c'est-à-dire ce qui attire notre attention, ce qui nous surprend. Il est :

[un type de signe ou de représentation] qui renvoie à son objet non pas tant parce qu'il a quelque similarité ou analogie avec lui ni parce qu'il est associé avec les caractères généraux que cet objet se trouve posséder, que parce qu'il est en connexion dynamique (y compris spatiale) et avec l'objet individuel d'une part et avec les sens ou la mémoire de la personne pour laquelle il sert de signe, d'autre part. (Peirce 158)

La découverte de la petite barrette à l'effigie d'une grenouille bleue surprend. Même Furfooz demeure incapable d'expliquer son état de surprise. L'indice pour Quignard 
opère un déplacement supplémentaire en tant qu'il constitue une forme survivante d'inscription inconsciente, de trace mnésique, c'est-à-dire de rebuts de mémoire. Il n'est pas un simple rapport de contiguïté. Il possède une couche d'équivoque supplémentaire dans la mesure où sa signification est dissimulée et semble a priori incompréhensible. On ne pourrait le déchiffrer spontanément. Sa structure est celle des survivances, des choses qui reviennent, qui resurgissent de l'inconscient, qui resurgissent de vestiges cristallisés. Il y a donc un mouvement qui appelle à une incessante reconfiguration des habitudes, à un déplacement constant où la dimension matérielle du signe se dissout, où le visible est constamment imprégné d'une forme d'inquiétude, d'une étrangeté.

L’objet-rebut excède son champ phénoménal. Dans l'œuvre de Quignard, son apparition dans le monde visible dissimule systématiquement une «part maudite» invisible. Au même titre que le Jadis constitue une temporalité qui «précède l'apparition du spectateur dans le visible » et «qui se caractérise par sa non-visibilité » (Sur le jadis 161), le rebut est un objet susceptible de contenir des parts d'invisibilité, c'est-à-dire des fragments du Jadis. Comme Vincent Giraud le souligne : «toute vision est par nature déceptive et décevante. Le drame de tout voir est de n’être que ce mouvement qui nous porte vers l'invisible comme vers ce qui ne peut, par essence, être vu» (290). Le regard d'Édouard Furfooz ne peut pleinement s'emparer de l'objet auquel il fait face : il y a toujours une part qui demeure inaccessible. L'acte de voir dépasse ce qui est donné dans le visible : tant l'objet que le sujet sont marqués par une forme d'inquiétude. DidiHuberman écrit : «l'acte de donner à voir n'est pas l'acte de donner des évidences visibles à des paires d'yeux qui se saisissent unilatéralement du “don visuel” pour s'en 
satisfaire unilatéralement. Donner à voir, c'est toujours inquiéter le voir, dans son acte, dans son sujet» (Ce que nous voyons 51$)$. La réflexion que nous offre Quignard sur le rebut et le monde des objets se veut en réalité une réflexion sur la subjectivité, c'est-àdire sur la mémoire de la personne pour laquelle le rebut fait signe. Simultanément, le rebut excède cette logique d'opposition binaire entre objet et sujet pour déborder dans un champ de vestiges beaucoup plus anciens.

Le rebut est donc un indice qui a une fonction de représentation partielle. Il remplace une absence, mais cette absence demeure en quelque sorte indicible et innommable. «Force est de constater, écrit Vincent Giraud, que la ruine du visible, à laquelle s'affrontent les personnages de Quignard, reste le plus souvent solidaire d'une traque éperdue qui veut à tout prix sauver du visible ce qui pourrait être tenu pour un vestige de l'invisible» (291). Premièrement, le déchet, comme résidu matériel, comme trace d'un reste en ruine, agit donc comme un relais entre le monde phénoménal et le monde invisible, entre le temps proprement humain et social et le temps originaire du Jadis, entre le monde de la conscience et celui de l'inconscience. Il est un objet paradoxal qui permet d'échapper au monde des objets et de dévoiler un autre monde marqué par l'absence et le perdu. Deuxièmement, ce type de signe fait l'objet d'une traque, d'une chasse. Les traces pour Quignard «ne sont donc jamais visibles en tant que traces. Elles ne sont visibles que si elles sont cherchées comme marques de ce qui n'est plus là. Toute trace est une bête absente, une chasse possible de ce qui ne s'y voit pas. Seule leur attente les découvre » (Sur le jadis 67). La signification des déchets non seulement est instable et partiellement accessible, mais elle doit faire l'attention d'une réceptivité toute particulière 
sur le mode de l'attente et du guet. Précisément, le geste du collectionneur consiste à sauver «en isolant par le court-circuit de l'usage, parmi les objets du monde certains vestiges en lesquels il croit retrouver le jadis » (Giraud 293).

Reste à comprendre la valeur indicative particulière des signes-rebuts dans Les escaliers de Chambord. La petite barrette en forme de grenouille indique une absence et une perte et cet élément, qui lance le développement de l'intrigue, est un jeu de piste que nous devons à présent suivre, comme l'antiquaire suit la piste des jouets. En effet, Les escaliers de Chambord est un roman de l'indice, un roman où le lecteur est appelé à déchiffrer, tout au long de la lecture, les signes laissés «en jachère » par Quignard; signes qui à la première lecture semblent indéchiffrables, mais qui dévoilent pleinement leur valeur indicative au fur et à mesure que l'intrigue se déploie.

\subsection{Des rebuts physiques aux rebuts de la mémoire}

La clef interprétative pour lire Les escaliers de Chambord réside dans une quête «sémiotique » organisée par le monde des jouets et des rebuts. Le lecteur est invité à effectuer un travail interprétatif visant à repérer les indices parsemés tout au long du roman : il est complice d'un jeu de traque, d'une chasse aux symptômes. Quignard érige un immense champ de signes qui résistent systématiquement à leur interprétation parce qu'ils sont volontairement ambigus et équivoques. Comme nous avons vu précédemment, ces signes-rebuts sont en réalité des traces qui dissimulent autre chose et qui pointent vers une perte et un manque plus originaire. Dans Les escaliers de Chambord, la perte originaire se déploie par diverses vagues de réminiscence. 
Nous avons déjà vu comment la petite barrette bleue en forme de grenouille occasionne la réminiscence d'un fond refoulé chez Édouard Furfooz, un fond marqué par sa propre indicibilité. Nous réalisons vers la fin du roman que cette petite barrette, qui en soi est un objet a-signifiant, dépourvu de fonction, pointe vers la perte d'une personne. La personne disparue resurgit lorsqu'Édouard fera l'acquisition d'un appartement proche du jardin du Luxembourg à Paris. Ce lieu revêt une grande importance à ses yeux, car il avait passé une partie de son enfance dans un appartement adjacent appartenant à sa tante. Alors qu'Édouard traverse le quartier, il réalise la chose suivante : «Traverser le jardin du Luxembourg, traverser son enfance, c'était une seule et même chose» (Escaliers 242). L'espace acquiert une fonction mémorative : l'espace rencontre le temps, il relie des moments disjoints dans le temps. L'espace devient une métonymie pour l'enfance, mais aussi une dimension susceptible de faire remonter ce qui précisément a été refoulé lors de cette période. Cette topique de la mémoire rétablit des durées qui furent abolies et les reconstruit anachroniquement. Gaston Bachelard écrit dans $L a$ poétique de l'espace: «[c]'est par l'espace, c'est dans l'espace que nous trouvons les beaux fossiles de durée concrétisés par de longs séjours. L'inconscient séjourne. Les souvenirs sont immobiles, d'autant plus solides qu'ils sont mieux spatialisés » (28). En parcourant l'espace Jadis habité, Furfooz tombe sur un fossile qui permet de revivifier ce qui fut oublié. Quignard décrit le travail de remémoration, alors que Furfooz flâne dans le jardin du Luxembourg, en ces termes :

Il avait l'impression qu'il cherchait depuis l'enfance quelque chose au bas des taillis, quelque chose de petit et de précieux, qu'il allait être grondé et peut-être mis à mort s'il ne le retrouvait pas plus tôt. Où il lui semblait qu'une main, au-dessus de lui, désignait quelque chose dans l'ombre qu'il 
ne voyait pas. Il en était mortifié. Il est vrai qu'une fois, jadis, traversant le jardin du Luxembourg, la petite condisciple de l'école de la rue Michelet avait montré du doigt un reflet de clarté dans les buissons. ... .

C'est ainsi qu'il se souvint une nouvelle fois qu'il avait aimé une petite fille, et qu'il découvrit une petite fille sans visage, sans mains, sans nom . . . il avait même une impression de cheveux mouillés, très mouillés — avec une barrette de bakélite ou de jade en forme de grenouille avait habité avenue de l'Observatoire. (Escaliers 243-244)

Cet épisode met fin aux déplacements frénétiques qui avaient jusqu'alors motivé la quête de Furfooz. Cette dernière, qui était animée principalement par une recherche insatiable d'objets et de jouets de collection, trouve finalement une forme de résolution alors qu'il achète l'appartement situé sur l'avenue de l'Observatoire, à deux pas de la résidence d'enfance d'une petite fille dont le souvenir demeure encore flou. L'espace est ici investi du pouvoir «de véhiculer une survivance» (Didi-Huberman, Génie du non-lieu 144). En parcourant ce lieu, de petits détails aux allures insignifiantes, par exemple les taillis qui jonchent le jardin, suscitent un ressouvenir, une remémoration, une mémoire involontaire pour le dire comme Marcel Proust, ${ }^{19}$ c'est-à-dire le retour d'une expérience qui était bel et bien perdue. Mais ce retour, bien qu'étant à portée de main, n'est pas encore pleinement accessible; il apparaît sous le mode de la hantise, du spectre. Furfooz se souvient de cette petite fille qu'il avait aimée, mais il reste toujours incapable de recoller l'entièreté des évènements refoulés. Néanmoins, il parvient à redonner une signification au rebut qu'il gardait soigneusement dans sa poche depuis le début du roman. Le

\footnotetext{
${ }^{19}$ Dans Du côté de chez Swann, Proust écrit : «Il en est ainsi de notre passé. C'est peine perdue que nous cherchions à l'évoquer, tous les efforts de notre intelligence sont peine inutile. Il est caché hors de son domaine et de sa portée, quelque objet matériel (en la sensation que nous donnerait cet objet matériel), que nous ne soupçonnons pas. Cet objet, il dépend du hasard que nous rencontrions avant de mourir, ou que nous ne rencontrions pas» (88). Il y a donc une conceptualisation analogue de la mémoire, dite involontaire, chez Quignard alors que le ressouvenir advient toujours par inadvertance.
} 
processus sémiosique ${ }^{20}$ trouve une certaine quiétude alors que ce signe, qui de prime abord semblait indéchiffrable, finit par indiquer ce pour quoi il prenait place.

Impossible à prononcer, le nom de la petite fille reste «sur le bout de la langue ». Nous savons à présent que Furfooz a perdu quelqu'un, mais le visage, les mains et le nom demeurent encore inaccessibles à la mémoire. Comment alors retrouver ce nom? La particularité de ce type d'oubli — car ici, Furfooz est bien conscient qu'il a oublié quelque chose - c'est que toute recherche «volontaire» bute contre l'incapacité de la conscience à organiser la mémoire et à en faire sens. Freud souligne dans Au-delà du principe de plaisir que nos processus psychiques ne sont pas «disposés dans l'ordre du temps, ... le temps ne leur fait subir aucune modification, ... [et] on ne peut pas leur appliquer la catégorie du temps» (70). Lorsque nous cherchons dans notre mémoire quelque chose d'oublié, tout y est désynchronisé, nos pensées ne sont jamais organisées dans le temps chronologique. En règle générale, plus nous tentons d'organiser notre pensée, plus l'élément oublié semble vouloir s'éloigner. Comme Quignard le souligne, «la fonction présente de la mémoire n'est pas celle du stockage. ... C'est de l'élection, du prélèvement, du rappel et du retour d'un unique élément au sein de ce qui a été stocké en bloc»(Le nom sur le bout de la langue, 66). Cette élection, de ce que renferme la mémoire, fonctionne plutôt comme surgissement, comme un geste involontaire marqué par une attention flottante et par la distraction ${ }^{21}$.

\footnotetext{
${ }^{20}$ Il est important de noter que dans la sémiotique peircienne, la sémiose renvoie à ce procès dynamique de signification visant à délimiter un interprétant final, c'est-à-dire une habitude interprétative. La sémiose est donc un processus imprévisible et dynamique permettant un déplacement des signes et une migration des significations. Voir Peirce, Charles Sanders. Écrits sur le signe, rassemblés, traduits et commentés par Gérard Deledalle. Paris : Éditions du Seuil, 1978.

${ }^{21}$ La notion de distraction, chère à Walter Benjamin, « est donc un mode inconscient de perception à l'état de veille ; il est réellement inconscient en ce qu'il est autonome par rapport à la conscience et remplit
} 
Cette hantise d'un passé insaisissable échappe systématiquement à la pleine reconstruction de la mémoire. Rétrospectivement, nous pouvons identifier dans Les escaliers de Chambord trois mouvements différents visant à «reconstruire» cette mémoire. L'intrigue du roman est basée sur une triple quête cachant des motifs inconscients : celle des relations et des intrigues amoureuses, celle du nom perdu et celle des jouets. Ces trois quêtes sont interdépendantes. Tout au long du roman, Furfooz aura de nombreuses rencontres amoureuses qui ne parviennent jamais à le combler. Il y a toujours quelque chose qui manque et chacune de ses relations semble plutôt exacerber une ineffable crise. À la fin du roman, alors que Furfooz voyage au-dessus de l'Atlantique, il commence à recoller les morceaux du casse-tête, alors que tout au long du roman, ces pièces ne semblaient offrir a priori aucune véritable image. Quignard écrit :

Dans l'avion qui le ramenait de New York à Paris il griffonna, durant des heures, des comptes. [. . .] Sur une feuille de papier, au milieu des colonnettes de chiffres, il notait ces noms qu'il évoquait: Francesca, Laurence... Il dessinait de vagues vagues, des silhouettes de branchages d'arbres. Il se dit : «Tout a commencé à se détraquer en septembre lorsque j'ai quitté Francesca !» Il s'arc-bouta et sortit la petite barrette bleue de sa poche. «Tout a commencé par ce petit bout de matière plastique. Persuadé que j'étais d'être poursuivi par une petite âme. J'étais une proie poursuivie par un secret....». (309)

Furfooz parvient à relier la petite barrette non seulement à une figure — infigurable — de son passé, mais réalise que celle-ci dissimule en réalité un autre traumatisme, originaire cette fois. Par distraction, il gribouille des vagues en les associant avec les noms des personnes qui hantent ses pensées quotidiennes et amoureuses. Quignard poursuit :

précisément des fonctions d'ordre réflexe là où la conscience est devenue inopérante, voire gênante.» (4) Voir l'article de Pascal Rousse, «Perception urbaine, distraction et stratification chez Benjamin, Eisenstein et Vertov », Appareil, Numéro spécial (2008). 
Il posa la barrette. ... Il regarda les noms qu'il avait notés machinalement, les prénoms des femmes dont il s'était dépris depuis à peine un an. Ses coups de passion étaient devenus de plus en plus instables et moins durables. La liste n'était pas infinie, perdue entre les branchages et les chiffres :

Francesca

Laurence

Ottilia

Roza

Adriana (310)

C'est ainsi que de façon inattendue, le nom sur le bout de la langue revient sous la forme d'un rébus - un rébus qui fut déclenché par la force sémiosique d'un rebut —, d'un acrostiche qui dévoile le nom oublié : Flora. Par la rétroaction du nom, convoqué par le langage, Furfooz parvient à «quitter le "pur présent" pour le "passé simple"» (Sur le jadis, 131), ou mutatis mutandis, à frapper le passé d'une actualité des plus brûlante. Le passé s'ouvre, permettant aux évènements refoulés de remonter, en un éclair, à la surface. La mémoire fait alors sentir ses «effets de retard» et son «action virulente de désynchronisation avec le réel » (Sur le jadis, 128).

Nous apprenons par la suite que Flora était bel et bien cette petite écolière qui habitait rue de l'Observatoire et qui avait été le premier amour d'enfance de Furfooz. Selon Laurent Demanze, le motif de la collection amoureuse dans Les escaliers de Chambord n'est pas une simple accumulation frénétique de conquêtes, mais davantage un parcours qui constitue un «chantier archéologique, où le sujet amoureux désenfouit au plus profond de soi les premières empreintes » (128). Cette «empreinte» originaire est celle d'une perte intense, la découverte du cadavre de la petite Flora, décédée par noyade, et qui plongea Furfooz dans une grave crise catatonique et amnésique. Ce n'est qu'à rebours que nous pouvons reprendre intégralement les thématiques et les intrigues du 
roman pour saisir comment les actions portées par Furfooz étaient toutes mues par la recherche inconsciente de ce fond traumatique. Quignard conclut son roman sur cette prise de conscience : «Il songea à ces noms qui lui avaient fait acheter un appartement, l'avaient séparé de deux femmes, ou les lui avaient fait aimer. A aucun moment il n'avait commandé son destin. Il était le jouet de quelques lettres. Il avait été le jouet de ses jouets mêmes » (Les Escaliers, 324). La quête de jouets qui traverse le roman apparaît à présent comme une quête secondaire. Par la dimension matérielle du langage, par ces «signifiants » qui renvoient aux objets de son désir, il parvient à toucher «à la fois à l'ordre du réel et à celui du symbolique » (Lapeyre-Desmaison, «Pascal Quignard : une poétique de l'agalma»49). Autrement dit, la langue permet de montrer ce qui est inaccessible, d'en dévoiler l'absence, sans néanmoins atteindre directement le Réel. Pour Lapeyre-Desmaison, cette dimension biface de la lettre est une «tentative d'enserrer la surprise de l'objet (ou l'objet comme surprise), comme ce qui ménage l'absence tout en la mettant en valeur» (49). Le langage pour Quignard est donc «la seule résurrection pour ce qui a disparu » (Sur le jadis 31), mais la résurrection, ou la trace de survivance, demeure toujours partielle. Elle est indicative sans être totalement rédemptrice : la perte reste définitive.

\subsection{L'enfance : retour vers le Jadis.}

L'intrigue du roman aboutit dans la divulgation du nom qui éclaire le double motif de l'accumulation qui traverse le roman. Tant la collection des objets que la collection des intrigues amoureuses apparaissent dès lors comme l'objet d'une perte, pointant vers un temps perdu, celui du «premier royaume» (Asselin 12). Ce premier 
royaume est celui l'enfance. La thématique de l'enfance chez Quignard doit être comprise comme un «paradigme élémentaire» mettant en jeu de nombreuses ambivalences. Bruno Blanckeman retrace ce motif dans l'ensemble des romans de Quignard. Il dit de l'enfance qu' «[e]lle est simultanément âge d'or — non parole, anticonscience, adhésion spontanée au monde ... - et âge de fer — temps des séquelles, des orientations irréversibles, des courbes creusées de la vie adulte»(Blanckeman 182). L'enfance, d'un côté, est ce temps à jamais perdu, ce temps d'avant la conscience, d'avant le langage ; mais simultanément, elle est une séquelle, elle est cette temporalité qui est radicalement présente en tant qu'elle remonte aux racines, aux origines. L'enfance est un passé dont la présence se fait toujours sentir : sa marque temporelle est donc essentiellement anachronique.

Dans Les escaliers de Chambord, nous avons évoqué l'importance de ce thème de diverses manières. Pour Chantal Leypre-Desmaison, chez Quignard, «le trait de l'enfance fait partie de l'esthétique romanesque d'ensemble» (116). Furfooz est prisonnier d'un monde inaccessible, et inconsciemment, il tente de s'en approcher par l'accumulation quasi névrotique de jouets, c'est-à-dire d'objets qui appartiennent à cet autre monde. Le même motif doit être découvert dans la recherche frénétique des relations amoureuses. Celles-ci sont d'une part un symptôme de cet amour «archaïque» qui doit être retrouvé, mais aussi des marques de l'enfance. L'amour que Furfooz porte pour le personnage de Laurence est une porte entr'ouverte vers ce temps. Quignard écrit : «Elle était comme inondée d'un rire plus vaste qu'elle et qui ne paraissait pas lui être personnel, qui s'abattait sur elle, crevait en elle comme une averse imprévisible. Elle 
ruisselait d'enfance. Quelque chose de jeune, d'invincible, fait d'une nouveauté invincible étincelait dans ses yeux alors » (Escaliers 99-100). L'enfance est le temps du rire, de l'imprévisible, de l'ouverture vers la nouveauté : elle a une force. C'est cette force originaire que Furfooz tente de retrouver parmi les autres personnages qui peuplent ce roman.

Finalement, le titre même du roman évoque l'enfance. Nous comprenons à la fin du roman que durant son enfance, Édouard avait visité le château de Chambord avec la petite Flora. ${ }^{22} \mathrm{Ce}$ lieu évoque directement l'enfance. Quignard écrit :

Il se remémorait seulement le grand escalier central, blanc, gigantesque s'élevant au centre du donjon. Il courait avec les autres enfants. . . Ils avaient aussi, hélas, pour préparer cette visite, une dictée interminable sur ces escaliers interminables. . . La dictée décrivait avec beaucoup de pédantisme et de nombreux termes techniques à l'orthographe impossible les deux montées conçues jadis par Léonard de Vinci autour du vide central, vertigineux - entre les fenêtres découpées où ses condisciples, une petite fille et lui-même se penchaient en criant et se montraient du doigt - et qui superposait leur révolution de telle sorte qu'on ne cessait jamais de voir l'autre sans le rencontrer jamais. ... Sans cesse on était abandonné de celui qu'on avait sous les yeux. (50-51)

Plusieurs éléments attirent ici notre attention. Le château comme structure apparaît vraisemblablement comme une structure enfantine, un espace de jeu qui suscite une excitation toujours nouvelle pour les enfants. On y monte et on y descend les escaliers en forme d'ADN; on rencontre l'autre pour le perdre immédiatement, en attendant la prochaine surprise, la prochaine apparition qui suscitera à son tour un nouvel émoi. On poursuit l'autre, mais il n'apparaît que momentanément, pour disparaître aussitôt : la

22 «Et il lui semblait qu'il avait oublié un nom. D'une façon comparable, quand il s'était trouvé face à l'escalier central, il avait été étonné de ne pas retrouver avec plus de chaleur ou de précision le souvenir qu'il avait conservé d'une visite qu'il avait faite enfant,. . . timide et crispée, avec tenant cérémonieusement, pour monter dans l'autocar la main d'une petite fille timide et crispée . . . .» (76) 
traque ne mène nulle part. Mais ce n'est qu'en regardant par les fenêtres découpées que l'autre apparaît, soit au-dessus, soit en dessous, mais toujours séparé par un vide abyssal et vertigineux. L'énigme du roman se trouve en quelque sorte condensée dans cette structure.

Dans un passage ultérieur, Quignard dira du château qu'il est la «[r]uine la plus blanche et la plus belle de France et qui n'avait jamais été que le chantier d'une ruine » (75), avec son allure blanchâtre, fantomatique et spectrale. Le château est un morceau central du casse-tête mémoriel — en ruine — que Quignard déploie. Il renvoie à différents moments de sa vie — à son enfance, à sa vie adulte — et à différents personnages rattachés à ces périodes, soit ceux de Flora et Laurence. En parlant de Laurence, Quignard écrit : «Ce prénom aimé tout neuf, tout naissant dans sa bouche, était comme ce château inhabité que sa tente Ottilia voulait rejoindre : la vie tout à coup pouvait surgir » (75-76). Ce surgissement est donc marqué du sceau de la nouveauté. À cet égard, Chantal Leypre-Desmaison remarque à quel point la thématique de l'enfance dans l'ensemble des romans de Quignard doit se concevoir comme un temps de l'énigme. «Tout d'abord, écrit-elle, l'enfant, dans ces romans, est bien cet habitant des frontières : il représente, au titre d'une image particulière chérie, le court-circuit entre ontogenèse et phylogenèse, puisqu'il est encore hors langage, proche des origines» (Mémoire de l'origine, 117). Figure habitant sur le seuil, il vacille entre deux mondes. Le privilège de l'enfant, qui n'a pas encore été n'envahit pas le langage, est d'être encore capable de voir la nouveauté dans le monde (Les escaliers de Chambord, 314). L'enfant est naissance, encore proche de son animalité, encore proche de l'origine : il est la personnification du 
Jadis. Tous ces éléments du roman ont donc une solidarité mystérieuse guidée par la «main » de l'enfant. En ce sens, Les escaliers de Chambord est bel et bien un roman du Jadis. 


\section{CONCLUSION}

De qui et de quoi Quignard est-il le contemporain ? Cette question, que nous avons laissée en suspend depuis le début du présent travail, peut à présent trouver une forme de résolution. L'œuvre de Quignard, comme nous l'avons souligné, est profondément en rupture avec son temps et convoque des éléments hétéroclites renvoyant à différentes époques, à différents schèmes de pensée et représentations du monde. Quignard rode tel un spectre entre les périodes historiques : il convoque les fantômes du passé pour les faire réapparaitre dans le temps présent. C'est en partie ici que réside l'anachronisme propre à Quignard : il démontre que le temps présent est en perpétuel déphasage et que le passé a aussi cette dimension imperfective susceptible de revenir hanter le présent. Les concepts de survivance et de symptôme illustrent parfaitement ce qui est à l'œuvre dans le Dernier Royaume et dans Les escaliers de Chambord. Ils permettent de remonter la trace d'un passé enfoui dans la mémoire. Comme notre premier chapitre l'a démontré, le passé gît, patiente, attend d'être réveillé. Il est en perpétuelle tension entre présence et absence, entre l'oubli et la mémoire ; parfois, nous le côtoyons sans même nous en apercevoir. Les survivances et les symptômes sont ces indices qui nous permettent de rendre présent — à nouveau — ce fond absent et de rendre à portée de main ce qui semblait a priori inaccessible à la conscience.

La conceptualisation du jadis est solidaire de cette démarche anachronique visant à retrouver la trace d'un fond perdu inaccessible. Le jadis, comme Quignard le souligne, est ce temps de l'origine marqué, sur le plan ontologique, par son ouverture. Lorsque 
Quignard aborde le passé, il ne le fait pas comme un historien : il ne recherche pas des faits, des vérités factuelles, ou encore des explications causales qui pourraient expliquer le temps présent. Il l'aborde comme un antiquaire, comme un collectionneur, comme un monteur. Comme nous l'avons mentionné dans notre deuxième chapitre, l'écriture fragmentaire du Dernier Royaume implique un travail esthétique de montage visant à relier des figures, des moments et des temps hétérogènes. Le travail de montage quignardien est implicitement solidaire d'une réflexion sur la temporalité. Le fragment jouit d'un rôle fondamental, celui de re-monter ce que l'histoire officielle avait rejeté et de dévoiler un fond. Le Jadis est ce fond qui émerge par le travail de montage des fragments du passé. Il est ce temps de l'archaïque, de l'Arkhè, de l'origine; un temps inaccessible, mais qui ne cesse de survenir.

L'infigurable Jadis réapparaît dans le roman Les escaliers de Chambord, cette fois-ci incarné dans le personnage de la petite Flora. La perte traumatique de cet amour de jeunesse épouse la structure du jadis : cette scène restera infigurable et inaccessible au personnage d'Édouard Furfooz tout au long du roman. Le roman illustre cette survenance du Jadis par divers procédés qui relèvent d'une sémiotique de l'indice ou d'un paradigme indiciaire, pour le dire comme Carlo Ginzburg. ${ }^{23}$ Telle est la méthode qui permet de redonner une présence à ce qui est absent et définitivement perdu. La figure du collectionneur incarnée par Furfooz accomplit ce travail de découverte et de liaisons entre «les fragments » abandonnés dans le passé. L’homologie entre le travail de Quignard dans le Dernier Royaume et celle de Furfooz est ici frappante : les deux insufflent un sens

${ }^{23}$ Voir entre autres Ginzburg, Carlo. «Signes, traces, pistes. Racines d'un paradigme de l'indice. ». Le Débat (1980) : 3-44. 
nouveau à ces matériaux qui ont été délaissés. Les jouets du collectionneur ont une valeur indiciaire et indicative particulière : ils pointent vers l'enfance, ils relient passé et présent, ils remontent l'origine et déplace les usages, les contextes. La même fonction est partagée par le rôle des rebuts : ils doivent revivifier ce qui fut oublié. C'est ce rôle qui fut octroyé par Quignard à la petite barrette bleue en forme de grenouille. Cet objet-déchet est une marque de survivance qui convoque des régions éloignées de la mémoire, mais il est aussi un symptôme qui déchire le monde phénoménal et qui déplace les significations.

Pour Quignard, comme le souligne Simon Saint-Onge, «[1]'intérêt porté à l'objet sordide ou au déchet est redevable au Perdu qu'il couve et qui est prêt à surgir par la force du Jadis » (163). Ce «Perdu», incarné dans la figure de Flora, resurgit par la force du Jadis, c'est-à-dire, comme nous l'avons vu dans notre deuxième chapitre, par cette dynamique rythmique entre le moment fort de la perte et celui de la revenance. Édouard Furfooz est comme cet Infans freudien qui rejoue inlassablement la perte de sa mère en lançant sa petite bobine attachée à un fil ; il «joue» avec ses jouets et avec ses rebuts, sans savoir que ceux-ci «couvent» une perte fondamentale et un temps perdu. Le rebut expose ce temps inaccessible à la mémoire. Nous retrouvons ici cette distinction entre le passé et le Jadis. Le passé est complété, la mort de la petite Flora est advenue, rien ne pourra empêcher cet événement d'avoir été : c'est un «passé-à-jamais » (Sur le jadis 33) irréversible. En opposition, le Jadis est ce qui survient et détermine toujours le présent sous le mode de l'ouverture : la quête des jouets, la quête amoureuse, sont des marques de ce passé «qui n'a pas encore fini de surgir » (Sur le jadis 149). Dans cette perspective, les quêtes de Furfooz sont marquées par un passé toujours ouvert, c'est-à-dire un passé 
libéré de sa séquence chronologique. Le Jadis n'est pas l'événement traumatique en luimême, mais il est cette force qui permet à ce fond de revenir hanter le présent : il est l'événement traumatique dans son perpétuel devenir. D'où toute l'importance de la thématique de l'enfance dans les romans de Quignard et dans sa conceptualisation du Jadis. L'enfance est proche de l'origine, mais une origine en devenir. Toutes ces empreintes de l'enfance qui ponctuent et rythment l'intrigue romanesque sont des portes entr'ouverte dans lesquels peut s'immiscer le Jadis avec sa force mystérieuse. « Jadis est un enfant qui joue comme un enfant»(77), nous dit Quignard dans un fragment de Sordidissimes. Le pays des jouets, le pays des enfants, est aussi le pays du Jadis. L'enfance est un temps inaccessible qui hante la vie adulte tel un spectre, mais qui ouvre simultanément un vivant futur. ${ }^{24}$

\footnotetext{
${ }^{24}$ Dans Spectre de Marx, Jacques Derrida écrit : «Le propre du spectre, s'il y en a, c'est qu'on ne sait pas s'il témoigne en revenant d'un vivant passé ou d'un vivant futur, car le revenant peut marquer déjà le retour du spectre d'un vivant promis. Intempestivité encore, et désajustement du contemporain » (qtd. dans Ruffel, Le dénouement 162).
} 


\section{BIBLIOGRAPHIE}

\section{Corpus étudié}

Quignard, Pascal. Abîmes (Dernier Royaume, Tome III), Paris : Gallimard, folio, 2002.

---. Le Nom Sur Le Bout De La Langue. Paris : P.O.L., 1993.

---. Les escaliers de Chambord. Paris : Gallimard, 1989.

---. Les Ombres errantes (Dernier Royaume, Tome I). Paris : Gallimard, folio, 2002.

---. Rhétorique spéculative. Paris : Calmann-Lévy, 1995.

---. Sordidissimes (Dernier Royaume, Tome V), Paris : Gallimard, folio, 2005.

---. Sur le jadis (Dernier Royaume, Tome II), Paris : Gallimard, folio, 2002.

---. Une gêne technique à l'égard des fragments. Paris : Fata Morgana, 1986.

\section{Littérature secondaire sur Pascal Quignard}

Alvares, Cristina. « Au commencement était le fauve. Prédation et fondement animal de l'humain chez Pascal Quignard. » Pascal Quignard. Translation et métamorphose. Ed. Mireille Calle-Gruber, Jonathan Degenève, Irène Fenoglio. Paris : Hermann, 2015. 219-232.

Asselin, Guillaume. «Du (dé)bris symbolique. », Protée, 36.1 (2008) : 7-15.

Blanckeman, Bruno. Les récits indécidables: Jean Echenoz, Hervé Guibert, Pascal Quignard. Villeneuve D’Ascq (Nord): Presses Universitaires Du Septentrion, 2000.

Bonnefis, Philippe, and Dolorès Lyotard. Pascal Quignard, Figures D'un Lettré. Actes du Colloque de Cerisy-la-Salle (août 2004). Paris : Galilée, 2005. 
Demanze, Laurent. «Collection érotique et vertige archaïque Les escaliers de Chambord de Pascal Quignard. » Litteratures Littératures 69 (2013) : 123-34.

Giraud, Vincent. «L'invisible et la proie. Une lecture de Pascal Quingard.» Studia Phaenomenologica VIII (2008): 283-306.

Gorrillot, Bénédicte. «P. Quignard ou le fragmentaire dialectique : au-delà du cut-up ?», Contemporary French and Francophone Studies, 18.3 (2014) : 258-265.

Lapeyre-Desmaison, Chantal. «Pascal Quignard : Une poétique de L'agalma. »Études Françaises 40.2 (2004) : 39-53.

---. Mémoires de l'origine : Un essai sur Pascal Quignard. Paris : Les Flohic Éditeurs, 2001.

Marchetti, Adriano. Pascal Quignard: La mise au silence. Seyssel : Champ Vallon, 2000.

Messager, Mathieu. «Dans la métamorphose, l'aoriste triomphe» Pascal Quignard. Translation et métamorphose. Ed. Mireille Calle-Gruber, Jonathan Degenève, Irène Fenoglio. Paris : Hermann, 2015. 205-218.

Motard-Noar, Martine. «Les Astérisques et "la fixité du regard perdu" chez Pascal Quignard », Littératures [Online], 72 (2015). Web. 27 février 2015.

Claudette Oriol-Boyer «Les escaliers de Chambord de Pascal Quignard. Roman d'apprentissage de la lecture et de l'écriture ». Pascal Quignard, figures d'un lettré, Actes du Colloque de Cerisy-la-Salle (août 2004). Ed. Philippe Bonnefis et Dolores Lyotard. Paris : Galilée, 2005. 147-164.

Pautrot, Jean-Louis. Pascal Quignard ou le fonds du monde. Amsterdam : Rodopi, 2007. 
Ravel, Agne. Les Lieux De Pascal Quignard: Actes Du Colloque De L'Université Du Havre, 29 Et 30 Avril 2013. Paris : Gallimard, 2014.

Rodrigues, Vinícius Dalben. «Pascal Quignard et l'âme ancrée dans l'intervalle », non plus, 4 (2013) : 121-130

Saint-Onge, Simon. «Le temps contemporain ou le jadis chez Pascal Quignard», Études françaises, 44.3 (2008) : 159-172.

Samoyault, Tiphaine et Quignard, Pascal. «Dialogue avec Pascal Quignard et Tiphaine Samoyault sur les catégories du temps. »Qu'est-ce que le contemporain. Ed. Lionel Ruffel. Nantes : Cécile Defaut, 2010. 199-236.

Samoyault, Tiphaine. «'Il Faut Se Souvenir Que Les Dinosaures N'apparurent Qu'en 1841” — Pascal Quignard Et L'histoire. »(Fabula / Colloques), Fabula / Les colloques (2013). Web. 15 novembre 2015.

Viart, Dominique, «Les "fictions critiques” de Pascal Quignard», Études françaises, $40: 2(2004): 25-37$.

Zhuo, Yue. «Le roman lieu sans terre ». Critique, 721-722 (2007) : 520-532

\section{Autres œuvres citées.}

-- « Anachronisme. » Le Grand Robert de la langue Française. 2e ed. 1992.

---. «Qu'est-ce que le contemporain » dans Nudités, Paris : Rivage Poche, 2009. 19-31

Agamben, Giorgio. Enfance et histoire: Destruction de l'expérience et origine de l’histoire. Paris : Payot, 1989. 
---. Stanze. Parole et fantasme dans la culture occidentale. Trad. Yves Hersant. Paris : Rivages poche, Petite Bibliothèque, 1998.

Bachelard, Gaston. La poétique de l'espace. Paris : PUF, coll. Quadrige, 1957.

Barthes, Roland, «Le discours de l'histoire. » Social Science Information, 6 (1967) : 6375.

Benjamin, Walter. Euvres III. Trad. Maurice de Gandillac, Rainer Rochlitz et Pierre Rusch. Paris : Gallimard, Folio, 2000.

---. Charles Baudelaire. Un poète lyrique à l'apogée du capitalisme. Paris. Petite Bibliothèque Payot, 2002.

---. Origine du drame baroque allemand. Trad. Sibylle Muller. Paris : Flammarion, 1985.

---. Paris, capitale du XIXe siècle : Le livre des Passages. Paris : Cerf, 1989.

Blanchot, Maurice. L'entretien infini. Paris : Gallimard, 1969.

Bonvarlet, Soizic. «Walter Benjamin, ou la possibilité de ne pas trahir l'enfance. » Variations Variations 8 (2006) : 50-58. Web. 14 avril 2016.

Deleuze, Gilles. Logique du sens. Paris : Éditions de minuit, 1969.

Didi-Huberman, Georges. Atlas ou le gai savoir inquiet. L'œil de l'histoire, 3. Paris : Éditions de minuit, 2011.

---. Ce que nous voyons, ce qui nous regarde. Paris : Éditions de Minuit, 1992.

---. Devant le temps. Histoire de l'art et anachronisme des images. Paris : Éditions de minuit, 2000.

---. Génie Du Non-lieu : Air, Poussière, Empreinte, Hantise. Paris : Éditions de Minuit, 2001. 
---. Génie du non-lieu. Air, poussière, empreinte, hantise. Paris : Éditions de Minuit, 2001.

---. L'Image survivante. Histoire de l'art et temps des fantômes selon Aby Warburg. Paris : Éditions de Minuit, 2002.

Freud, Sigmund. «Au-delà Du Principe De Plaisir». Essais de psychanalyse. Paris : Petite bibliothèque Payot, 1981. 41-115.

---. Métapsychologie. Trad. Jean Laplanche et J.-B. Pontalis. Paris : Gallimard, Folio, 1968.

Ginzburg, Carlo. «Signes, traces, pistes. Racines d'un paradigme de l'indice. » Le Débat (1980) : 3-44.

Marcel Proust, À la recherche du temps perdu. Du côté de chez Swann, Paris, Gallimard, 1988.

Méchoulan, Eric. Pour une histoire esthétique de La Littérature. Paris: Presses Universitaires de France, 2004.

Nietzsche, Friedrich. Considérations inactuelles. Paris : Gallimard, 1990.

Peirce, Charles Sanders. Écrits sur le signe, rassemblés, traduits et commentés par Gérard Deledalle. Paris : Éditions du Seuil, 1978.

Pomian, Krzysztof. «Histoire culturelle, histoire des sémiophores.» Pour une histoire culturelle. Ed. Jean-Pierre Rioux et Jean-François Sirinelli. Paris : Seuil, 1997. 73-100.

Proust, Marcel. À la recherche du temps perdu. Du côté de chez Swann. Paris : Gallimard, 1988. 
Rancière, Jacques. «Le concept d'anachronisme et la vérité de l'historien. » L'Inactuel, 6 (1996) : 53-68.

Ricœur, Paul. La mémoire, l'histoire, l'oubli. Paris : Éditions du Seuil, 2000.

---. Temps et récit 1. L'intrigue et le récit historique. Paris : Éditions du Seuil, 1983.

Robin, Régine. La Mémoire saturée. Paris : Stock, 2003.

Rousse, Pascal. «Perception urbaine, distraction et stratification chez Benjamin, Eisenstein et Vertov », Appareil, Numéro spécial (2008). Web. 20 Avril 2016.

Ruffel, Lionel. Le Dénouement. Paris : Verdier, coll. «Chaoïd », 2005. 